\title{
Invariant Spherical Hyperfunctions on The Tangent Space of A Symmetric Space
}

\author{
Jiro Sekiguchi
}

\section{Introduction}

This paper deals with the study of invariant spherical hyperfunctions on the tangent space of a semisimple symmetric space.

First recall the results on invariant eigendistributions (=IED) on a real semisimple Lie algebra. Hence let $g$ be a real semisimple Lie algebra and let $g_{r s}$ be the totality of regular semisimple elements of $g$. Then Harish-Chandra showed the following famous theorem (cf. [HC1]):

Theorem I. Every invariant eigendistribution on $\mathrm{g}$ is a locally $L^{1}$-function.

At one step of the proof of this theorem, he also showed the following theorem ([HC3]):

Theorem II. Let $u$ be an IED on $\mathrm{g}$. Then the restriction of $u$ to $\mathfrak{g}_{r s}$ determines $u$ itself, that is, if $u$ is zero on $g_{r s}$, then $u$ is identically zero on the whole space $\mathrm{g}$.

In this paper, we attempt to obtain a generalization of the results mentioned above for invariant spherical hyperfunctions on the tangent. To explain our main result, we need some notation. Let $\sigma$ be an involution of $\mathfrak{g}$ and let $\mathfrak{g}=\mathfrak{h}+\mathfrak{q}$ be the direct sum decomposition for $\sigma$, where $\mathfrak{h}$ and $q$ are the 1 and -1 eigenspaces of $\sigma$, respectively. Then one can naturally define an invariant spherical hyperfunction $(=\mathrm{ISH})$ on the vector space $\mathfrak{q}(\S 5)$. An ISH is, by definition, a hyperfunction solution of a holonomic system $\mathscr{M}_{A}$ of differential equations on $\mathfrak{q}$. In the case where $\mathrm{g}=\mathrm{g}^{\prime} \oplus \mathfrak{g}^{\prime}\left(\mathfrak{g}^{\prime}\right.$ is semisimple) and $\sigma(X, Y)=(Y, X)((X, Y) \in \mathfrak{g})$, every ISH on $\mathfrak{q}\left(\simeq \mathfrak{g}^{\prime}\right)$ is nothing but an IED on $\mathfrak{g}^{\prime}$. Hence the notion of an ISH is a natural generalization of that of an IED on a semisimple Lie algebra.

Since every ISH $u$ on $\mathfrak{q}$ is real analytic on $\mathfrak{q}_{r s}$ (=the set of $\mathfrak{q}$-regular semisimple elements of $q$ ), it is rather easy to treat the restriction of $u$ to 
$\mathfrak{q}_{r s}$. This and Theorem II above lead us to the following problem:

Problem. Does there exist a non-zero ISH $u$ on $\mathfrak{q}$ such that Supp $(u)$ is contained in $\mathfrak{q}-\mathfrak{q}_{r s}$ ?

An ISH $u$ is called singular if $\operatorname{Supp}(u)$ is contained in $\mathfrak{q}-\mathfrak{q}_{r s}$. Then there exists a non-zero singular ISH on $\mathfrak{q}$ for some symmetric pair $(\mathfrak{g}, \mathfrak{h})$ (cf. §6). But we can show the following theorem which is an analogue of Theorem II (cf. §5, Theorem 5.2).

Theorem 1. Let $(\mathfrak{g}, \mathfrak{h})$ be a symmetric pair. Assume that $\delta_{\mathfrak{q}}(Z)>0$ for every $\mathfrak{q}$-distinguished element $Z$ of $\mathfrak{q}$ (for the definition of the number $\delta_{\mathrm{q}}(Z)$, see $\left.\S 1\right)$. Then there exists no non-zero singular invariant spherical hyperfunction on $\mathfrak{q}$.

Our method of the proof of Theorem 1 is based on Atiyah's lecture note [A] which is an intelligent introduction to Theorem I of HarishChandra. We are going to explain the main part of the argument. Let $\square$ be the pseudo-Laplacian on $\mathfrak{q}$. Following Atiyah [A], for an arbitrary element $Z$ of $q$, we calculate the radial component of $\square$ at $Z$ by use of a specified local coordinate system near $Z$. The choice of this local coordinate system plays a fundamental role in the proof of Theorem 1 . In the case where $Z$ is nilpotent, the radial component of $\square$ for the local coordinate is already obtained by van Dijk [vD]. At any rate, if $\delta_{\mathrm{a}}(Z)>0$ for an arbitrary $\mathfrak{q}$-distinguished element $Z$ of $\mathfrak{q}$, the proof of Theorem 1 goes parallel to the arguments in [A]. So we obtain Theorem 1.

Needless to say, the assumption of Theorem 1 holds for a Riemannian symmetric pair or a pair of the form $\left(\mathfrak{g}^{\prime} \oplus \mathfrak{g}^{\prime}, \mathfrak{g}^{\prime}\right.$ ) (which is regarded as the case of the Lie algebra $g^{\prime}$ ). But for these cases, the conclusion of Theorem 1 is already known. In fact, in the former case, every ISH on $q$ is real analytic. On the other hand, Theorem 1 is reduced to Theorem II in the latter case.

The next problem is to determine whether for a given symmetric pair $(\mathfrak{g}, \mathfrak{h})$, the assumption of Theorem 1 holds or not. Hence it is important to classify all the symmetric pairs satisfying the assumption. Let $\left(\mathfrak{g}_{c}, \mathfrak{h}_{c}\right)$ be the complexification of $(\mathfrak{g}, \mathfrak{h})$ and let $\mathfrak{g}_{0}$ be a real form of $\mathfrak{g}_{c}$ such that $\mathfrak{f}_{0}=\mathfrak{h}_{c} \cap \mathfrak{g}_{0}$ is a maximal compact subalgebra of $\mathfrak{g}_{0}$. We shall show the next theorem in Section 6 (cf. Theorem 6.3).

Theorem 2. Retain the notation above. Let $\Sigma$ be the restricted root system of $\mathrm{g}_{0}$. For every root $\lambda \in \Sigma$, let $m_{\lambda}$ be the multiplicity of $\lambda$. Assume that $m_{\lambda}+m_{2 \lambda} \leq 2$ for all $\lambda \in \Sigma$. (For example, this condition holds in the case where $\mathfrak{g}_{0}$ is a normal real form of $\mathfrak{g}_{c}$.) Then the assumption of Theorem 
1 holds for the pair $(\mathfrak{g}, \mathfrak{h})$.

Our proof of this theorem is based on the classification of all the nilpotent orbits of complex simple Lie algebras.

At an early stage of the preparation of this paper, the author proved Theorem 1 only in the case of invariant spherical distribution (=ISD). T. Oshima showed him Proposition 2.3 in Section 2 as well as its proof. Once the proposition is established, so is Theorem 1 not only for the case of distributions but also for the case of hyperfunctions. It is worthwhile to state a conjecture concerning the difference between ISD and ISH. Let $\mathscr{M}_{A}$ be the holonomic system introduced in Section 5. Note that an ISD is, by definition, a distribution solution of $\mathscr{M}_{1}$.

Conjecture. $\mathscr{M}_{A}$ is a regular holonomic system in the sense of Kashiwara-Kawai (cf. [HK]).

If this is true, one would show, as a corollary, that any ISH turns out to be an ISD. Hence, there exists no difference between ISH and ISD.

Acknowledgement. The author wishes his hearty thanks to Mr. T. Kawazoe for fruitful discussions with him about this topic and also to Prof. T. Oshima for showing him the proof of Proposition 2.3.

\section{§1. Semisimple and nilpotent elements associated with symmetric spaces}

In this section, we discuss the structure of the tangent space of a symmetric space. This will be needed in the subsequent discussions.

(1.1) First introduce some standard notation. In this paper, $\mathbf{N}$ always means the set $\{0,1,2, \cdots\}$. If $V$ is a real vector space, we denote by $V_{c}$ its complexification. Also we denote by $V^{*}$ and $V_{c}^{*}$ the duals of $V$ and $V_{c}$, respectively. Moreover $S\left(V_{c}\right)$ denotes the symmetric algebra over $V_{c}$. For a real Lie algebra $\mathfrak{g}, U\left(\mathfrak{g}_{c}\right)$ denotes the enveloping algebra of $\mathfrak{g}_{c}$ and for an element $X$ of $\mathfrak{g}$ and a linear subspace $V$ of $\mathfrak{g}$, we put $V_{X}=$ $\{Z \in V ;[Z, X]=0\}$.

(1.2) Let $g$ be a real semisimple Lie algebra and let $\sigma$ be its involution. As usual, $\mathfrak{h}$ and $\mathfrak{q}$ denote the 1 and -1 eigenspaces of $\sigma$, respectively. Then $\mathfrak{g}=\mathfrak{h}+\mathfrak{q}$ is a direct sum decomposition. In this paper, the pair $(\mathfrak{g}, \mathfrak{h})$ obtained in this way is called a symmetric pair. If $G$ is the adjoint group of $g$, then $\sigma$ is lifted to $G$. For the sake of simplicity, the lifting of $\sigma$ is denoted by the same letter. Define $G^{\sigma}=\{g \in G ; \sigma g=g\}$ and let $H$ be the identity component of $G^{\sigma}$. Then $\mathfrak{h}$ is the Lie algebra of $H$. Since $[\mathfrak{h}, \mathfrak{q}] \subset \mathfrak{q}, H$ acts on $\mathfrak{q}$. The coset space $G / H$ is a semisimple symmetric 
space and the tangent space of $G / H$ at $e H$ is identified with $q$.

Let $\mathscr{S}$ and $\mathscr{N}$ be the totality of semisimple elements and that of nilpotent elements of $\mathfrak{g}$, respectively. Put $\mathscr{S}(\mathfrak{q})=\mathscr{S} \cap \mathfrak{q}$ and its elements are called semisimple elements of $\mathfrak{q}$. Similarly, put $\mathscr{N}(\mathfrak{q})=\mathscr{N} \cap \mathfrak{q}$ and its elements are nilpotent elements of $\mathfrak{q}$.

(1.3) First recall the Jordan decomposition of elements of $q$.

Lemma 1.1. Take $Z_{0} \in \mathfrak{q}$.

(i) There exist unique elements $A_{0} \in \mathscr{S}(\mathfrak{q})$ and $X_{0} \in \mathscr{N}(q)$ such that $Z_{0}=A_{0}+X_{0}$ and that $\left[A_{0}, X_{0}\right]=0$.

(ii) $\mathfrak{g}_{Z_{0}}=\mathfrak{g}_{A_{0}} \cap \mathfrak{g}_{X_{0}}$.

Definition 1.2. (i) The number $\min \left\{\operatorname{codim}_{q} H \cdot Z ; Z \in \mathfrak{q}\right\}$ is called the rank of $(\mathfrak{g}, \mathfrak{h})$ or that of $\mathfrak{q}$ and is denoted by rank $\mathfrak{q}$.

(ii) As element $Z$ of $\mathfrak{q}$ is called $\mathfrak{q}$-regular if $\operatorname{codim}_{\mathfrak{q}} H \cdot Z$ conicides with rank q.

Lemma 1.3. Let $S\left(\mathfrak{q}_{c}\right)^{H}$ be the totality of H-invariant elements of $S\left(\mathfrak{q}_{c}\right)$. Then there exist homogeneous elements $P_{1}, \cdots, P_{l}(l=$ rank $q)$ such that $S\left(\mathfrak{q}_{c}\right)=\mathbf{C}\left[P_{1}, \cdots, P_{l}\right]$.

This is due to Chevalley (cf. [KR]).

(1.4) We review semisimple elements of $\mathfrak{q}$. A linear subspace $\mathfrak{a}$ of $\mathfrak{q}$ is called a Cartan subspace of $\mathfrak{q}$ if $\mathfrak{a}$ is a maximal abelian subspace of $\mathfrak{q}$ consisting of semisimple elements. By definition, the dimension of $\mathfrak{a}$ coincides with rank $\mathfrak{q}$. It is known (cf. [OM]) that there are only a finite number of $H$-conjugate classes of Cartan subspaces of $\mathfrak{q}$. Now take a Cartan subspace $\mathfrak{a}$ of $\mathfrak{q}$ and fix it once for all. For a linear form $\lambda$ on $\mathfrak{a}_{c}$, we define

$$
\mathfrak{g}_{c}^{\lambda}=\left\{X \in \mathfrak{g}_{c} ;[A, X]=\lambda(A) X \quad \text { for all } A \in \mathfrak{a}_{c}\right\}
$$

and

$$
\Sigma(\mathfrak{a})=\left\{\lambda \in \mathfrak{a}_{c}^{*} ; \mathfrak{g}_{\mathfrak{c}}^{2} \neq\{0\}\right\}-\{0\} .
$$

Then $\Sigma(\mathfrak{a})$ becomes a root system. In fact, $\Sigma(\mathfrak{a})$ coincides with the restricted root system of an appropriate real form of $g_{c}$ (cf. [OS]). Define $\mathfrak{a}^{\prime}=\{A \in \mathfrak{a} ; \lambda(A) \neq 0$ for all $A \in \mathfrak{a}\}$. Then it is clear that every element of $\mathfrak{a}^{\prime}$ is $\mathfrak{q}$-regular. For this reason, this is called a $\mathfrak{q}$-regular semisimple element of $\mathfrak{a}$.

Let $a_{1}, \cdots, a_{k}$ be the totality of representatives of mutually not $H$ conjugate Cartan subspaces of $\mathfrak{q}$. Put $\mathfrak{q}^{\prime}=\bigcup_{i=1}^{k} \bigcup_{h \in H} h \cdot \mathfrak{a}^{\prime}$. Then $\mathfrak{q}^{\prime}$ 
consists of $\mathfrak{q}$-regular elements and is Zariski dense in $\mathfrak{q}$. Note that not every $\mathfrak{q}$-regular element of $\mathfrak{q}$ is contained in $\mathfrak{q}^{\prime}$. For example, as will be stated later, in some cases, there exist $\mathfrak{q}$-regular nilpotent elements in $\mathfrak{q}$ (cf. (1.9)).

(1.5) Take a semisimple element $A_{0}$ of $\mathfrak{q}$. Then its centralizer $z=$ $\mathfrak{g}_{A_{0}}$ is reductive and is $\sigma$-invariant. Let $\mathfrak{c}$ be its center and let $z_{s}$ be its semisimple part. Now put $\mathfrak{c}^{-}=\mathfrak{c} \cap \mathfrak{q}, z^{+}=z \cap \mathfrak{h}, z_{s}^{+}=z_{s} \cap \mathfrak{h}, z^{-}=z \cap \mathfrak{q}$ and $z_{s}^{-}=z_{s} \cap q$. Then $z_{s}=z_{s}^{+}+z_{s}^{-}$is a direct sum and $\left(z_{s}, z_{s}^{+}\right)$is a symmetric pair.

Definition 1.4. The pair $\left(\partial_{s}, \partial_{s}^{+}\right)$thus obtained is called a sub-symmetric pair of $(\mathfrak{g}, \mathfrak{h})$.

(1.6) Next we review nilpotent elements of $q$. The following lemmas are fundamental in the subsequent discussion (cf. [KR], [vD]).

Lemma 1.5. (i) $H$ leaves $\mathscr{N}(q)$ invariant and there are only a finite number of $H$-orbits of $\mathscr{N}(\mathfrak{q})$.

(ii) $\mathscr{N}(\mathfrak{q})=\left\{X \in \mathfrak{q} ; P(X)=P(0)\right.$ for all $\left.P \in S\left(\mathfrak{q}_{c}\right)^{H}\right\}$.

Remark 1.6. It is known (cf. $[\mathrm{KR}]$ ) that $\operatorname{codim}_{\mathrm{q}} \mathscr{N}(\mathfrak{q}) \leq l=\operatorname{rank} \mathfrak{q}$. But the equality does not hold in general. In fact, if $(\mathfrak{g}, \mathfrak{h})$ is Riemannian, then $\mathscr{N}(\mathfrak{q})$ consists of only one element 0 and therefore $\operatorname{codim}_{\mathfrak{q}} \mathscr{N}(\mathfrak{q})=$ $\operatorname{dim} \mathfrak{q}<l$.

Lemma 1.7. Let $X_{0} \in \mathscr{N}(\mathfrak{q})$. Then there exist $A_{0} \in \mathfrak{h}$ and $Y_{0} \in \mathfrak{q}$ such that

$$
\left[A_{0}, X_{0}\right]=2 X_{0}, \quad\left[A_{0}, Y_{0}\right]=-2 Y_{0}, \quad\left[X_{0}, Y_{0}\right]=A_{0} \text {. }
$$

Definition 1.8. Retain the notation in Lemma 1.7. Then $\left(A_{0}, X_{0}, Y_{0}\right)$ is called a normal $S$-triple and the Lie algebra $\Upsilon=\mathbf{R} A_{0}+\mathbf{R} X_{0}+\mathbf{R} Y_{0}$ is a TDS.

By definition, $\mathfrak{l}$ is isomorphic to $\mathfrak{g l}(2, \mathbf{R})$. Let $\theta_{0}$ be a Cartan involution on $\mathfrak{l}$ defined by $\theta_{0}:\left(A_{0}, X_{0}, Y_{0}\right) \rightarrow\left(-A_{0},-Y_{0},-X_{0}\right)$. It is clear that $\theta_{0}$ commutes with $\sigma \mid$ l.

Lemma 1.9 ([vD]). $\quad \theta_{0}$ can be extended to a Cartan involution $\theta$ on $\mathfrak{g}$ which commutes with $\sigma$.

(1.7) Let $\left(A_{0}, X_{0}, Y_{0}\right)$ be a normal $S$-triple. The centralizer $\mathfrak{g}_{x_{0}}$ is left invariant by ad $A_{0}$ and also by $\sigma$. This implies that $\mathfrak{g}_{x_{0}}=\mathfrak{h}_{x_{0}}+\mathfrak{q}_{x_{0}}$ is a direct sum and ad $A_{0}$ leaves $\mathfrak{h}_{x_{0}}$ and $\mathfrak{q}_{x_{0}}$ invariant. Noting this, we can 
take a basis $w_{1}, \cdots, w_{r}$ of $\mathfrak{q}_{X_{0}}$ which are eigenvectors of ad $A_{0}\left(r=\operatorname{dim} \mathfrak{q}_{X_{0}}\right)$. Hence $\left[A_{0}, w_{i}\right]=n_{i} w_{i}$ for a number $n_{i}(1 \leq i \leq r)$. Then each $n_{i}$ is a nonnegative integer (cf. [vD]).

Definition 1.10. Define $\delta_{q}\left(X_{0}\right)=\sum_{i=1}^{r}\left(n_{i}+2\right)-\operatorname{dim} \mathfrak{q}$.

It is clear from the definition that $\delta_{q}\left(h \cdot X_{0}\right)=\delta_{q}\left(X_{0}\right)$ for all $h \in H$.

(1.8) Let $B($,$) be the Killing form on \mathrm{g}$. Then we write $\omega(X)=$ $B(X, X)(X \in \mathfrak{q})$ and call $\omega$ the Casimir polynomial on $\mathfrak{q}$. It is clear from the definition that $\omega$ is an $H$-invariant non-degenerate quadratic form on $\mathfrak{q}$.

Definition 1.11. $X_{0}$ is $\mathfrak{q}$-distinguished nilpotent if $X_{0}$ does not commute with any non-zero semisimple element of $\mathfrak{q}$.

Lemma 1.12 ([vD]). The following conditions on $X_{0}$ are mutually equivalent.

(i) $X_{0}$ is $\mathfrak{q}$-distinguished nilpotent.

(ii) $\omega(X)=0$ for all $X \in \mathfrak{q}_{X_{0}}$.

(iii) $\omega(X)=0$ for all $X \in \mathfrak{q}_{Y_{0}}$.

(iv) $n_{i}>0(1 \leq i \leq r)$.

(v) $\mathfrak{q}_{X_{0}} \cap \mathfrak{q}_{Y_{0}}=0$

(1.9) A nilpotent element $X_{0} \in \mathfrak{q}$ is called $\mathfrak{q}$-regular if $\operatorname{dim} \mathfrak{q}_{X_{0}}=$ rank $\mathfrak{q}(=l)$, or equivalently, $\operatorname{codim}_{\mathfrak{q}} H \cdot X_{0}=l$ (cf. Definition 1.2).

If each of $\mathfrak{g}$ and $\mathfrak{h}$ is a normal real form of a complex semisimple Lie algebra, then $\mathscr{N}(\mathfrak{q})$ actually contains $\mathfrak{q}$-regular nilpotent elements. But, in some cases, this does not occur. A typical example is the case of Riemannian symmetric pairs (cf. Remark 1.6).

Let $X_{0} \in \mathfrak{q}$ be $\mathfrak{q}$-regular nilpotent. Then $X_{0}$ has the following properties (cf. [KR], [vD]):

(i) $\operatorname{dim} \mathfrak{q}_{X_{0}}=l$.

(ii) If $n_{1}, \cdots, n_{l}$ are the integers defined as in (1.7), one may take the homogeneous generators $P_{1}, \cdots, P_{l}$ of $S\left(\mathfrak{q}_{c}\right)^{H}$ in such a way that $n_{i}+2=2 \operatorname{deg} P_{i}(i=1, \cdots, l)$.

(iii) $X_{0}$ is $\mathfrak{q}$-distinguished.

(iv) $\delta_{q}\left(X_{0}\right)=l+\sum_{\lambda \in \Sigma^{+},(1 / 2) \lambda \notin \Sigma}\left(2-m_{\lambda}-m_{2 \lambda}\right)$.

(Here $\Sigma$ is the root system defined as in (1.4) and $\Sigma^{+}$is a positive system of $\Sigma$.)

(1.10) Let $Z_{0} \in \mathfrak{q}$ and let $Z_{0}=A_{0}+X_{0}$ be as in Lemma 1.1. Since $A_{0}$ is semisimple, we can define the sub-symmetric pair $\left(\partial_{s}, \partial_{s}^{+}\right)$and the vector spaces $\jmath_{s}^{-}, \mathfrak{c}^{-}$, as we did in (1.5). By definition, $X_{0}$ is a nilpotent element 
of $z_{s}^{-}$. Hence the number $\delta_{z_{s}}\left(X_{0}\right)$ is defined similarly. Then we put $\delta_{q}\left(Z_{0}\right)=\delta_{z s}-\left(X_{0}\right)$.

Definition 1.13. $Z_{0}$ is $\mathfrak{q}$-distinguished if $X_{0}$ is $z_{s}^{-}$-distinguished nilpotent.

If $Z_{0} \in \mathfrak{q}$ is $\mathfrak{q}$-regular, then $Z_{0}$ is $\mathfrak{q}$-distinguished. In fact, if $Z_{0}=A_{0}+$ $X_{0}$ is the Jordan decomposition of $Z_{0}$ as above, then $X_{0}$ is $z_{s}^{-}$-regular nilpotent.

(1.11) Let $A$ be a $\mathfrak{q}$-regular semisimple element of $\mathfrak{q}$. Then $\mathfrak{g}_{A}$ is left invariant by $\sigma$. So $\mathfrak{g}_{A}=\mathfrak{h}_{A}+\mathfrak{q}_{A}$ is a direct sum. Note that $\mathfrak{a}=\mathfrak{q}_{A}$ is a Cartan subspace of $\mathfrak{q}$ and that $\mathfrak{h}_{A}$ is the centralizer of $\mathfrak{a}$ in $\mathfrak{h}$. Then it easily follows that the number $m=\operatorname{dim} a-\operatorname{dim} \mathfrak{h}_{A}$ does not depend on the choice of $A$.

Lemma 1.14 (cf. $[\mathrm{KR}]$ ). $\quad \operatorname{dim} \mathfrak{q}_{X}-\operatorname{dim} \mathfrak{h}_{X}=m$ for all $X \in \mathfrak{q}$.

Corollary 1.15. $\operatorname{dim} \mathfrak{q}_{X}=\frac{1}{2}\left(\operatorname{dim} \mathfrak{g}_{X}+m\right)$ for all $X \in \mathfrak{q}$.

\section{$\S 2$. Preliminaries from differential equations}

(2.1) Let $M$ be a connected open subset of $\mathbf{R}^{m}$ containing the origin and $x=\left(x_{1}, \cdots, x_{m}\right)$ its Cartesian coordinate system. First introduce some standard notation. As usual, put $D_{x_{i}}=\partial / \partial x_{i}(i=1, \cdots, m)$. For any multi-index $\alpha=\left(\alpha_{1}, \cdots, \alpha_{m}\right) \in \mathbf{N}^{m},|\alpha|=\alpha_{1}+\cdots+\alpha_{m}$. Moreover, $x^{\alpha}=x_{1}^{\alpha_{1}} \cdots x_{m}^{\alpha_{m}}, D_{x}^{\alpha}=D_{x_{1}}^{\alpha_{1}} \cdots D_{x_{m}}^{\alpha_{m}}$. Let $\mathscr{A}(M)$ and $\mathscr{B}(M)$ denote the set of analytic functions on $M$ and that of hyperfunctions on $M$, respectively. Moreover, $\mathscr{B}_{M}$ denotes the sheaf of hyperfunctions on $M$.

In this paper, differential operators always mean those having analytic functions as coefficients. Let $P\left(x, D_{x}\right)$ be a differential operator defined on $M$. Then $P$ is expressed as follows:

$$
P=\sum_{\alpha \in N^{m,|\alpha| \leq d}} a_{\alpha}(x) D_{x}^{\alpha} .
$$

If $a_{\alpha}(x)$ is not identically zero for some $\alpha(|\alpha|=d)$, then $d$ is called the order of $P$ and is denoted by ord $P$. Moreover, $\sigma(P)(x, \xi)=\sum_{|\alpha|=d} a_{\alpha}(x) \xi^{\alpha}$ is called its principal symbol, where $\xi=\left(\xi_{1}, \cdots, \xi_{m}\right)$ is the conormal variable and $\xi^{\alpha}=\xi_{1}^{\alpha_{1}} \cdots \xi_{m}^{\alpha_{m}}$. For any $x_{0} \in M, P\left(x_{0}, D_{x}\right)$ is called the local expression of $P$ at $x_{0}$.

Define, for any $p \in S\left(\mathbf{C}^{m}\right)$, a differential operator $\partial(p)$ on $\mathbf{R}^{m}$ as follows. First, if $v=\left(v_{1}, \cdots, v_{m}\right) \in \mathbf{R}^{m}$, then define the vector field $\partial(v)$ on $\mathbf{R}^{m}$ by

$$
(\partial(v) f)(x)=\left.\frac{d}{d t} f(x+t v)\right|_{t=0},
$$


where $f(x)$ is an arbitrary analytic function on $\mathbf{R}^{m}$. If $v_{1}, \cdots, v_{k} \in \mathbf{R}^{m}$, then $\partial\left(v_{1} \cdots v_{k}\right)=\partial\left(v_{1}\right) \cdots \partial\left(v_{k}\right)$. Extending $\partial$ linearly to the symmetric algebra $S\left(\mathbf{C}^{m}\right)$, we have thus defined $\partial(p)$ for any $p \in S\left(\mathbf{C}^{m}\right)$. It is clear from the definition that for every $p \in S\left(\mathbf{C}^{m}\right)$, the local expression of $\partial(p)$ at every point of $\mathbf{R}^{m}$ coincides with itself.

(2.2) Let $N$ be a connected open subset of $\mathbf{R}^{n}$ containing the origin and $y=\left(y_{1}, \cdots, y_{n}\right)$ as its Cartesian coordinate. We always identify $N$ with a subset $\{0\} \times N$ of $M \times N$. The next lemma is fundamental (cf. [SKK]).

Lemma 2.1. Let $u(x, y)$ be a hyperfunction on $M \times N$. If $u(x, y)$ satisfies the differential equations

$$
D_{x_{i}} u=0(i=1, \cdots, m),
$$

then $u(x, y)$ is constant with respect to the variable $x$, that is $\left.u(x, y)\right|_{x=0}=v(y)$ is a well-defined hyperfunction on $N$ and $u(x, y)=v(x)$ holds on $M \times N$.

(2.3) In the rest of this section, we give propositions which play fundamental roles in the proof of Theorem 5.2 in Section 5 .

Proposition 2.2. Let $P\left(x, y, D_{x}, D_{y}\right)$ be a differential operator of order 2 defined on $M \times N$ and let $u(x, y)$ be a hyperfunction solution of the differential equation $P u=0$. Assume the following conditions: $y \in N$.

(i) $\sigma(P)(0, y, \xi, 0)$ is a non-degenerate quadratic form of $\xi$ for all

(ii) $\operatorname{Supp}(u) \subset N$.

Then $u=0$.

This follows from Chap. III, Prop. 2.1.3 in [SKK].

Proposition 2.3. Let $P$ be a differential operator defined on $M \times N$ of order 2 and of the following form:

$$
\begin{aligned}
P= & \sum_{j=1}^{m} a_{j}(x, y) D_{x_{1}} D_{x_{j}}+b(x, y) D_{x_{1}}+R_{1}\left(x, y, D_{x}^{\prime}, D_{y}\right) x_{1} D_{x_{1}} \\
& +R_{0}\left(x, y, D_{x}^{\prime}, D_{y}\right),
\end{aligned}
$$

where $D_{x}^{\prime}=\left(D_{x_{2}}, \cdots, D_{x_{m}}\right)$. Assume that

$a_{1}(x, y)=\lambda_{1} x_{1}$,

$a_{j}(x, y)=\lambda_{j} x_{j}+($ terms of order $\geq 2$ with respect to $x)$,

$b(x, y)=\mu+($ terms of order $\geq 1$ with respect to $x)$,

$\sigma\left(R_{0}\right)\left(0, y, \xi_{2}, \cdots, \xi_{m}, 0\right)=0$, 
where each $\lambda_{j}$ is a constant such that $\operatorname{Re} \lambda_{j}>0$ and $\mu$ is also a constant.

If $u(x, y)$ is a hyperfunction on $M \times N$ satisfying that

$$
P u=0, \quad \text { Supp }(u) \subset N,
$$

then $u(x, y)$ is expressed of the form

$$
u(x, y)=\sum f_{\alpha}(y) \delta^{(\alpha)}(x) \quad \text { (finite sum), }
$$

where each $f_{\alpha}(x)$ is a hyperfunction on $N$.

Remark. T. Oshima has shown the author the proof of Proposition 2.3. So he expresses his hearty thanks to T. Oshima.

Proof. Let $Z$ be an open subset of $\mathbf{C}^{m}$ such that $Z \cap \mathbf{R}^{m}=M$. Similarly, let $W \subset \mathbf{C}^{n}$ be an open set such that $W \cap \mathbf{R}^{n}=N$. Identify $W$ with the subset $\{0\} \times W$ of $Z \times W$. Let $\mathcal{O}_{Z \times W}$ be the sheaf of holomorphic functions on $Z \times W$ and put $\mathscr{J}=\sum_{j=1}^{m} \mathcal{O}_{Z \times W} x_{j}$. It follows from the definition that $\left(P x_{1}\right) \mathscr{J}^{d} \subseteq \mathscr{J}^{d}$ for any integer $d>0$.

To prove the proposition, we need a lemma.

Lemma 2.4. Choose a positive integer $h$ such that

$$
\operatorname{Re}\left(\frac{h}{m} \lambda_{j}+\mu\right)>0 \quad(j=1, \cdots, m) .
$$

Then for any open neighbourhood $U$ of the origin $0 \in Z \times W$, there exists a small open neighbourhood $V \subset U$ of 0 satisfying the conditions (i), (ii):

(i) $P x_{1}: \mathscr{J}^{n}(V) \rightarrow \mathscr{J}^{h}(V)$ is injective.

(ii) For any $f \in \mathscr{J}^{n}(U)$, there exists $a u \in \mathscr{J}^{n}(V)$ such that $P x_{1} u=f$ holds on $V$.

Assuming this lemma for a moment, we continue the proof of Proposition 2.3. Note that the adjoint $P^{*}$ of $P$ also satisfies the assumption of the proposition. Let $T$ be a sufficiently small complex neighbourhood of the origin and let $K$ be a compact subset of $T \cap N$. Replacing each point of $K$ with the origin and applying Lemma 2.4, we find that

$$
P^{*} x_{1}: \mathscr{J}^{h}(K) \rightarrow \mathscr{J}^{h}(K)
$$

is a topological automorphism as a DFS-space. Here $h$ is a sufficiently large integer satisfying the conditions (i), (ii) of Lemma 2.4 and

$$
\begin{aligned}
\mathscr{J}^{n}(K)=\frac{\lim _{U}}{\mathscr{J}^{n}(U)} & (U \text { runs through complex neighbourhoods } \\
& \text { of } K) .
\end{aligned}
$$


Note that $h$ is independent of the choice of $K$. Now put

$$
\mathscr{S}_{h}=\left\{u \in \mathscr{B}_{M \times N} ; f u=0 \text { for all } f \in \mathscr{J}^{h}\right\} .
$$

Since $\mathscr{S}_{h} \simeq \sum_{|\alpha|<h} \mathscr{B}_{N} \delta^{(\alpha)}(x), \mathscr{S}_{h}$ is regarded as not only a sheaf on $M \times N$ but also that on $N$. Taking the dual of the isomorphism (2.3.1), we obtain the isomorphism

$$
H_{K}^{0}\left(M \times N, \mathscr{B}_{M \times N}\right) \bmod \Gamma\left(K, \mathscr{S}_{h}\right) \stackrel{x_{1} P}{\longleftarrow} H_{K}^{0}\left(M \times N, \mathscr{B}_{M \times N}\right) \bmod \Gamma\left(K, \mathscr{S}_{h}\right) .
$$

Define the presheaf $\mathscr{F}$ on $M \times N$ as follows. For every open subset $D$ of $M \times N, \Gamma(D, \mathscr{F})=H_{D \cap N}^{0}\left(D, \mathscr{B}_{M \times N}\right) \bmod \Gamma\left(D, \mathscr{S}_{h}\right)$. Since both $\mathscr{B}_{M \times N}$ and $\mathscr{S}_{h}$ are flabby sheaves, we find that $\mathscr{F}$ becomes a flabby sheaf. Hence, if $D$ is contained in $T$, it follows that

$$
\mathscr{F}(D) \stackrel{x_{1} P}{\longleftarrow} \mathscr{F}(D)
$$

is an isomorphism. This implies the proposition.

Proof of Lemma 2.4. First introduce a partial order on $\mathbf{N}^{m}$. Let $\alpha=\left(\alpha_{1}, \cdots, \alpha_{m}\right), \alpha^{\prime}=\left(\alpha_{1}^{\prime}, \cdots, \alpha_{m}^{\prime}\right) \in \mathbf{N}^{m}$. We define that $\alpha<\alpha^{\prime}$ if and only if one of the conditions (a), (b) holds:

(a) $\alpha_{1}<\alpha_{1}^{\prime}$.

(b) $\alpha_{1}=\alpha_{1}^{\prime}$ and $|\alpha|<\left|\alpha^{\prime}\right|$.

Consider the differential equation

$$
\left(P x_{1}\right) u=f .
$$

Assume that $f(x, y)$ and $u(x, y)$ are formal power series of the forms

$$
\begin{aligned}
& f(x, y)=\sum_{\alpha \in \mathbb{N}^{m}, \beta \in \mathbb{N}^{n},|\alpha| \geq h} f_{\alpha \beta} x^{\alpha} y^{\beta} \quad\left(f_{\alpha \beta} \in \mathbf{C}\right) \\
& u(x, y)=\sum_{\alpha \in \mathbb{N}^{m, \beta} \sum_{\mathbb{N}^{n},|\alpha| \geq h} u_{\alpha \beta} x^{\alpha} y^{\beta}}\left(u_{\alpha \beta} \in \mathbf{C}\right) .
\end{aligned}
$$

Note that if $u(x, y)$ is of the form (2.3.4), then $f=P x_{1} u$ is expressed of the form (2.3.3). In the sequel, we always assume that $f$ and $u$ are the ones as in (2.3.3) and (2.3.4). Put

$$
\begin{aligned}
P_{0} & =\left(\sum_{j=1}^{m} \lambda_{j} x_{j} D_{x_{1}} D_{x_{j}}+\mu D_{x_{1}}\right) x_{1} \\
& =\left(x_{1} D_{x_{1}}+1\right)\left(\sum_{j=1}^{m} \lambda_{j} x_{j} D_{x_{j}}+\mu\right) .
\end{aligned}
$$


Then (2.3.2) is rewritten of the form

$$
P_{0} u=\left(P_{0}-P x_{1}\right) u+f \text {. }
$$

Comparing the coefficients of $x^{\alpha} y^{\beta}$ of both sides in (2.3.5), we find that

$$
\begin{aligned}
& \text { (left hand side })=\left(\alpha_{1}+1\right)\left(\sum_{j=1}^{m} \lambda_{j} \alpha_{j}+\mu\right) u_{\alpha \beta} \\
& \text { (right hand side) }=f_{\alpha \beta}+\sum_{\substack{\alpha^{\prime} \prime<\alpha \\
\left|\alpha^{\prime}\right|+\left|\beta^{\prime}\right| \leq|\alpha|+|\beta|+2}} c_{\alpha^{\prime} \beta^{\prime} \beta^{\prime}} u_{\alpha^{\prime} \beta^{\prime}},
\end{aligned}
$$

where $c_{\alpha^{\prime} \beta^{\prime}}^{\alpha \beta}$ are constants independent of the choices of $f$ and $u$. From the choice of $h$, we find that $\operatorname{Re}\left(\sum_{j=1}^{m} \lambda_{j} \alpha_{j}+\mu\right)>0$ for all $\alpha \in \mathbf{N}^{m},|\alpha| \geq h$. It follows from (2.3.5) and (2.3.6) that $u(x, y)$ is uniquely determined by $f(x, y)$.

We are going to prove by the method of majorants that if $f$ is convergent, then the formal power series solution $u$ of (2.3.2) is actually convergent. First we replace the coordinate $(x, y)$ with $\left(x_{1}^{3}, x_{2}, \cdots, x_{m}, y\right)$ and rewrite this by $(x, y)$ for the sake of simplicity. Then $\left(R_{1} x_{1} D_{x_{1}}+R_{0}\right) x_{1}$ is changed into the form

$$
\begin{array}{r}
x_{1}\left\{\widetilde{R}_{1}\left(x, y, x_{1} D_{x}^{\prime}, x_{1} D_{y}\right) x_{1} D_{x_{1}}+\widetilde{R}_{0}\left(x, y, x_{1} D_{x}^{\prime}, x_{1} D_{y}\right)\right\} \\
\quad=x_{1}\left\{\sum_{\alpha \in N^{m-1}, \beta \in N^{n},|\alpha|+|\beta| \leq 2} e_{\alpha \beta}(x, y)\left(x_{1} D_{x}^{\prime}\right)^{\alpha}\left(x_{1} D_{y}\right)^{\beta}\right\} .
\end{array}
$$

Here $\left(x_{1} D_{x}^{\prime}\right)^{\alpha}=x_{1}^{|\alpha|} D_{x_{2}}^{\alpha_{2}} \cdots D_{x_{m}}^{\alpha_{m}}$, etc, and each $e_{\alpha \beta}(x, y)$ is an analytic function. Moreover $\lambda_{1}$ is changed into $3 \lambda_{1}$. For the sake of simplicity, we rewrite this by $\lambda_{1}$. Put $s=x_{2}+\cdots+x_{m}$ and $t=y_{1}+\cdots+y_{n}$, and choose positive constants $\varepsilon, C, C^{\prime}>0$ satisfying the conditions

$$
\begin{gathered}
\varepsilon|\alpha| \leq\left|\sum_{j=1}^{m} \lambda_{j} \alpha_{j}+\mu\right| \quad\left(\forall \alpha \in \mathbf{N}^{m},|\alpha| \geq h\right) \\
a_{j}(x, y)-\lambda_{j} x_{j} \ll \frac{\varepsilon C^{\prime}\left(x_{1}+s\right)^{2}}{C-x_{1}-s-t} \quad(j=2, \cdots, m) \\
b(x, y)-\mu \ll \frac{C^{\prime}\left(x_{1}+s\right)}{C-x_{1}-s-t} \\
f(x, y) \ll \frac{C^{\prime}\left(x_{1}+s\right)^{h}}{C-x_{1}-s-t} \\
e_{\alpha \beta}(x, y) \ll \frac{C^{\prime}}{C-x_{1}-s-t} \quad \text { for all }(\alpha, \beta) .
\end{gathered}
$$


Put $\hat{f}(x, y)=C^{\prime}\left(x_{1}+s\right)^{n} /\left(C-x_{1}-s-t\right)\left(=\sum \hat{f}_{\alpha \beta} x^{\alpha} y^{\beta}\right)$ and let $x_{1} \hat{R}$ be the differential operator which is obtained from the one in (2.3.7) by changing each coefficient $e_{\alpha \beta}(x, y)$ with $C^{\prime} /\left(C-x_{1}-s-t\right)$, that is, $\hat{R}$ is defined by

$$
\hat{R}=\frac{C^{\prime}}{C-x_{1}-s-t} \sum_{\alpha, \beta}\left(x_{1} D_{x}^{\prime}\right)^{\alpha}\left(x_{1} D_{y}\right)^{\beta} .
$$

Moreover define

$$
\begin{aligned}
& \hat{P}_{0}=\varepsilon\left(x_{1} D_{x_{1}}+1\right)\left(\sum_{j=1}^{m} x_{j} D_{x_{j}}\right) \\
& \hat{S}=\frac{C^{\prime}\left(x_{1}+s\right)}{C-x_{1}-s-t}\left\{\varepsilon\left(x_{1}+s\right)\left(\sum_{j=2}^{m} x_{j} D_{x_{j}}\right)+1\right\}\left(x_{1} D_{x_{1}}+1\right)+x_{1} \hat{R}
\end{aligned}
$$

and consider the differential equation

$$
\left(\hat{P}_{0}-\hat{S}\right) \hat{u}=\hat{f} .
$$

Then, by means of an argument similar to the above one, one finds that for the given $\hat{f}(x, y)$, there exists a unique formal power series $\hat{u}(x, y)=$ $\sum_{|\alpha| \geq h} \hat{u}_{\alpha \beta} x^{\alpha} y^{\beta}$ satisfying (2.3.10). In particular, as in (2.3.6), we obtain the relations

$$
\varepsilon|\alpha|\left(\alpha_{1}+1\right) \hat{u}_{\alpha \beta}=\hat{f}_{\alpha \beta}+\sum \hat{c}_{\alpha^{\prime} \beta}^{\alpha \beta} \hat{u}_{\alpha^{\prime} \beta^{\prime}}
$$

Comparing (2.3.6) and (2.3.11), we find that $\hat{u}(x, y)$ is a majorant of $u(x, y)$. On the other hand, one easily shows that $\hat{u}(x, y)$ is expressed as a power series of $x_{1}, s, t$. Noting this, we may assume that $\hat{u}(x, y)=v\left(x_{1}, s, t\right)$ for a power series $v\left(x_{1}, s, t\right)$ of $x_{1}, s, t$. Then, from the definition, we find that

$$
\begin{aligned}
& \hat{P}_{0} \hat{u}=\varepsilon\left(x_{1} D_{x_{1}}+1\right)\left(x_{1} D_{x_{1}}+s D_{s}\right) v, \\
& \hat{S} \hat{u}=\left[\frac{C^{\prime}\left(x_{1}+s\right)}{C-x_{1}-s-t}\left\{\varepsilon\left(x_{1}+s\right) s D_{s}+1\right\}\left(x_{1} D_{x_{1}}+1\right)+x_{1} R^{\prime}\right] v,
\end{aligned}
$$

where $R^{\prime}=\left(C^{\prime} /\left(C-x_{1}-s-t\right)\right) \sum_{\alpha, \beta}\left(x_{1} D_{s}\right)^{|\alpha|}\left(x_{1} D_{t}\right)^{|\beta|}$ (cf. (2.3.9)). Then (2.3.10) turns out to be

$$
\left(P_{0}^{\prime}-S^{\prime}\right) v=\hat{f}
$$

where $P_{0}^{\prime}$ and $S^{\prime}$ are defined by

$$
\begin{aligned}
& P_{0}^{\prime}=\varepsilon\left(x_{1} D_{x_{1}}+1\right)\left(x_{1} D_{x_{1}}+s D_{s}\right) \\
& S^{\prime}=\frac{C^{\prime} s}{C-x_{1}-s-t}\left\{\left(\varepsilon s^{2} D_{s}+1\right)\left(x_{1} D_{x_{1}}+1\right)\right\} .
\end{aligned}
$$




$$
+x_{1}\left\{R^{\prime}+\frac{\varepsilon C^{\prime}\left(x_{1}+2 s\right)}{C-x_{1}-s-t} s D_{s}\left(x_{1} D_{x_{1}}+1\right)+\frac{C^{\prime}}{C-x_{1}-s-t}\left(x_{1} D_{x_{1}}+1\right)\right\}
$$

At this stage, we consider the differential equation

$$
\left(P_{0}^{\prime}-S^{\prime}\right) v=g \text {. }
$$

As in the previous case, assume that $g\left(x_{1}, s, t\right)$ and $v\left(x_{1}, s, t\right)$ are power series of the forms

$$
\begin{aligned}
& g\left(x_{1}, s, t\right)=\sum_{i+j \geq h} g_{i j k} x_{1}^{i} s^{j} t^{k} \\
& v\left(x_{1}, s, t\right)=\sum_{i+j \geq h} v_{i j k} x_{1}^{i} s^{j} t^{k} .
\end{aligned}
$$

If $g=\hat{f}$, then (2.3.13) is reduced to (2.3.12). For this reason, from now on, we treat (2.3.13) instead of (2.3.12). Then by an argument similar to the above one, it suffices to show that if $g\left(x_{1}, s, t\right)$ is convergent, so is the solution $v\left(x_{1}, s, t\right)$ of (2.3.13). To accomplish this, introduce the function $\eta\left(x_{1}, s, t\right)$ defined by

$$
\left(1-\frac{C^{\prime} s^{2}}{C-x_{1}-s-t}\right) \frac{\partial \eta}{\partial s}=\frac{C^{\prime} s}{C-x_{1}-s-t} \eta, \quad \eta\left(x_{1}, 0, t\right)=1 .
$$

Using $\eta$, we change the coordinates by $\left(x_{1}^{\prime}, s^{\prime}, t^{\prime}\right)=\left(x_{1}, \eta s, t\right)$ and rewrite the differential equation (2.3.13) into that of $x_{1}^{\prime}, s^{\prime}, t^{\prime}$. For the sake of simplicity, we exchange $x_{1}^{\prime}, s^{\prime}, t^{\prime}$ with $x_{1}, s, t$. Then (2.3.13) turns out to be

$$
\left[\left\{\varepsilon\left(3 x_{1} D_{x_{1}}+s D_{s}\right)+\phi\left(x_{1}, s, t\right)\right\}\left(x_{1} D_{x_{1}}+1\right)+x_{1} R^{\prime \prime}\right] v=g .
$$

where $\phi\left(x_{1}, s, t\right)$ is an analytic function defined in a neighbourhood of the origin, such that $\phi(0)=0$. In virtue of the discussion above, it suffices to show that if $g\left(x_{1}, s, t\right)$ is a convergent power series of the form (2.3.14), then the power series solution $v\left(x_{1}, s, t\right)$ of (2.3.17) is also convergent. We may assume that

$$
\phi \ll \hat{\phi}=\frac{x_{1}+s+t}{C-x_{1}-s-t}, \quad g \ll \hat{g}=\frac{C^{\prime}\left(x_{1}+s\right)^{h}}{C-x_{1}-s-t}
$$

holds for the constants $C, C^{\prime}>0$ and consider the differential equation

$$
\left\{\varepsilon\left(3 x_{1} D_{x_{1}}+1\right)\left(3 x_{1} D_{x_{1}}+s D_{s}\right)-\hat{\phi}\left(3 x_{1} D_{x_{1}}+1\right)-x_{1} \hat{R}^{\prime \prime}\right\} \hat{v}=\hat{g},
$$

where $\hat{R}^{\prime \prime}$ is a differential operator constructed from $R^{\prime \prime}$ in the same way obtaining $\hat{R}$ from $\left(R_{1} x_{1} D_{x_{1}}+R_{0}\right) x_{1}$. Then we find that $\hat{v}\left(x_{1}, s, t\right)$ is a 
majorant of $v\left(x_{1}^{3}, s, t\right)$. On the other hand, the differential equation (2.3.19) has regular singularities along the hypersurface $\left\{x_{1}=0\right\}$ (cf. [O]). Then in virtue of [O], we conclude that $\hat{v}\left(x_{1}, s, t\right)$ is convergent.

We have thus proved that $\hat{v}(x, y)=v\left(x_{1}, s, t\right)$ is convergent and therefore that the solution $u(x, y)$ of (2.3.2) is convergent. This implies (i) and (ii). In the above discussions, we assumed that $m>1$. But the case $m=1$ is easier to prove than the case $m>1$. In fact, in this case, the original equation (2.3.2) has regular singularities.

q.e.d.

As a corollary of Proposition 2.3, we obtain the next one.

Proposition 2.5. Retain the notation and the assumption in Proposition 2.3. Moreover assume that

$$
\sum_{j=1}^{m} \lambda_{j}\left(\alpha_{j}+1\right) \neq b(0) \quad \text { for all } \alpha \in \mathbf{N}^{m} .
$$

Then $u=0$.

Proof. Let $u(x, y)$ be a non-zero hyperfunction on $M \times N$ such that $P u=0$ and that $\operatorname{Supp}(u) \subset N$. Then, by means of Proposition 2.3, we may assume from the first that $u(x, y)=\sum_{|\alpha| \leq d} f_{\alpha}(y) \delta^{(\alpha)}(x), f_{\alpha}(y) \neq 0$ for some $\alpha(|\alpha|=d)$.

Let $f(y)$ be an arbitrary hyperfunction on $N$ and take $\alpha \in \mathbf{N}^{m}$. Then it follows that

$$
\begin{aligned}
P\left(f(y) \delta^{(\alpha)}(x)\right)= & \left\{-\sum_{j=1}^{m} \lambda_{j}\left(\alpha_{j}+1\right)+\mu\right\} f(y) \delta^{\left(\alpha^{\prime}\right)}(x) \\
& +\sum_{\beta \in \mathbb{N}^{m}, \beta<\alpha^{\prime}} g_{\beta}(y) \delta^{(\beta)}(x),
\end{aligned}
$$

where $\alpha^{\prime}=\left(\alpha_{1}+1, \alpha_{2}, \cdots, \alpha_{m}\right)$ and each $g_{\beta}(y)$ is a hyperfunction on $N$. Noting (2.3.21), we conclude from the assumption (2.3.20) that $u=0$. This contradicts the assumption.

q.e.d.

\section{§3. Radial components of differential operators}

(3.1) Following [HC2] and [vD], we introduce some notation. For any $X \in \mathfrak{q}_{c}, L_{X}$ is the linear endomorphism of $S\left(\mathfrak{q}_{c}\right)$ defined by $L_{X}(p)=X p$. On the other hand, for any $X \in \mathfrak{h}_{c}, d_{X}$ is the unique derivation of $S\left(\mathfrak{q}_{c}\right)$ which coincides on $\mathfrak{q}_{c}$ with $\operatorname{ad}_{8 c}(X)$. Furthermore, for any $Y \in \mathfrak{q}_{c}$, define $\sigma_{Y}(X)=L_{[X, Y]}+d_{X}\left(X \in \mathfrak{h}_{c}\right)$. Then $\sigma_{Y}$ is a representation of $\mathfrak{h}_{c}$ on $S\left(\mathfrak{q}_{c}\right)$. We can extend $\sigma_{Y}$ to the representation of $U\left(\mathfrak{h}_{c}\right)$ on $S\left(\mathfrak{q}_{c}\right)$ and denote it by the same letter. For $Y \in \mathfrak{q}$, let $\Gamma_{Y}$ be the linear mapping of $U\left(\mathfrak{G}_{c}\right) \otimes S\left(\mathfrak{q}_{c}\right)$ 
to $S\left(\mathfrak{q}_{c}\right)$ defined by $\Gamma_{Y}(g \otimes p)=\sigma_{Y}(g) p\left(g \in U\left(\mathfrak{G}_{c}\right), p \in S\left(\mathfrak{q}_{c}\right)\right)$.

(3.2) Now take an element $X_{0}$ of $\mathfrak{q}$. Let $U$ be a linear subspace of $\mathfrak{q}$ such that $\mathfrak{q}=U+\left[X_{0}, \mathfrak{h}\right]$ is a direct sum. On the other hand, let $V$ be a linear subspace of $\mathfrak{h}$ such that $\mathfrak{h}=V+\mathfrak{h}_{X_{0}}$ is a direct sum. For any $u \in U$, define the linear mapping $\phi_{u}$ of $V \times U$ to $\mathfrak{q}$ by $\phi_{u}(v, w)=w+\left[X_{0}+u, v\right]$. Then $\phi_{0}$ is bijective. Noting this, we define $\zeta(u)=\operatorname{det}\left(\phi_{u} \circ \phi_{0}^{-1}\right)(u \in U)$ which is a polynomial on $U$ with respect to an arbitrary linear coordinate. For the linear subspace $V$ of $\mathfrak{h}, S\left(V_{c}\right)$ is naturally identified with the subspace of $S\left(\mathfrak{h}_{c}\right)$. Let $S_{d}\left(\mathfrak{h}_{c}\right)$ be the totality of homogeneous elements of degree $d$ in $S\left(\mathfrak{h}_{c}\right)$ for every $d \in \mathbf{N}$. Put $S_{d}\left(V_{c}\right)=S\left(V_{c}\right) \cap S_{d}\left(\mathfrak{h}_{c}\right)$. Let $\lambda$ be the symmetrization of $S\left(\mathfrak{h}_{c}\right)$ onto $U\left(\mathfrak{h}_{c}\right)$. Using $\lambda$, we define $\Im_{d}\left(V_{c}\right)=$ $\lambda\left(S_{d}\left(V_{c}\right)\right), \mathfrak{S}\left(V_{c}\right)=\lambda\left(S\left(V_{c}\right)\right)$ and $\mathfrak{S}_{+}\left(V_{c}\right)=\sum_{d \geq 1} \mathfrak{S}_{d}\left(V_{c}\right)$.

Lemmas 3.1-3.3 below are shown by arguments similar to Lemmas 8 and 9 in [HC2].

Lemma 3.1. Let $u \in U . \quad$ If $\zeta(u) \neq 0$, then $\Gamma_{X_{0}+u}$ is a bijective mapping of $\mathfrak{S}\left(V_{c}\right) \otimes S\left(U_{c}\right)$ onto $S\left(\mathfrak{q}_{c}\right)$.

Lemma 3.2. Take $p \in S\left(\mathfrak{q}_{c}\right)$. Then there exist a non-negative integer $r$ and a polynomial mapping $\gamma_{p}$ of $U$ to $S\left(V_{c}\right) \otimes S\left(U_{c}\right)$ such that $\Gamma_{X_{0}+u}\left(\gamma_{p}(u)\right)$ $=\zeta(u)^{r} p$ for any $u \in U$.

In the above lemma, a polynomial mapping is used in the following sense. Let $A$ and $B$ be vector spaces. Assume that $\operatorname{dim} A<\infty$. Then $p: A \rightarrow B$ is called a polynomial mapping if $p(a)=\sum_{i} f_{i}(a) v_{i}$ (finite sum), where $v_{i} \in B$ and each $f_{i}(a)$ is a polynomial on $A$ with respect to a linear coordinate system.

Now put $U^{\prime}=\{u \in U ; \zeta(u) \neq 0\}$.

Lemma 3.3. Fix $p \in S\left(\mathfrak{q}_{c}\right)$. Then for any $u \in U^{\prime}$, there exist unique elements $\alpha_{u}(p) \in S\left(U_{c}\right)$ and $\beta_{u}(p) \in \widetilde{S}_{+}\left(V_{c}\right) \otimes S\left(U_{c}\right)$ such that $p-\alpha_{u}(p)=$ $=\Gamma_{X_{0}+u}\left(\beta_{u}(p)\right)$. Moreover $d^{0} \alpha_{u}(p) \leq d^{0} p$. (Here $d^{0} q$ denotes the degree of q.)

Definition 3.4. $\alpha_{u}(p)$ is called the radial component of $p$ at $u$ (with respect to $\left.X_{0}, U, V\right)$.

(3.3) For any $p \in S\left(\mathfrak{q}_{c}\right)$, we define the differential operator $\partial(p)$ as in Section 2. On the other hand, for any $X \in \mathfrak{h}$, define a vector field $\tau(X)$ on $\mathfrak{q}$ as follows:

$$
(\tau(X) f)(Y)=\left.\frac{d}{d t} f(Y+t[Y, X])\right|_{t=0} \quad(Y \in \mathfrak{q}) .
$$


(Here $f(Y)$ is an arbitrary analytic function on $\mathfrak{q}$.)

Let $D$ be a differential operator on an open subset $\Omega$ of $q$. Then we define a differential operator $A(D)$ on the open subset $U^{\prime} \cap\left(\Omega-X_{0}\right)$ by the same way as in [HÇ2, p. 283] (see also [vD]).

Let $\mathscr{D}(\Omega)$ denote the ring of differential operators on $\Omega$ and let $\mathscr{T}(\Omega)$ be the left ideal of $\mathscr{D}(\Omega)$ generated by the vector fields $\tau(X)(X \in \mathfrak{h})$.

Definition 3.5. (i) A hyperfunction $u$ on $\Omega$ is locally invariant if $\tau(X) u=0$ for all $X \in \mathfrak{h}$.

(ii) A differential operator $D$ on $\Omega$ is locally invariant if $[\tau(X), D]$ $=0$ for all $X \in \mathfrak{h}$.

(3.4) Return to the situation in (3.2). Take a basis $u_{1}, \cdots, u_{m}$ of $U$ and let $x_{1}, \cdots, x_{m}$ be its coordinates. Similarly, take a basis $b_{1}, \cdots, b_{n}$ of $V$ and let $y_{1}, \cdots, y_{n}$ be the coordinate with respect to this $V$-basis.

Lemma 3.6. Define the analytic mapping $F$ of $\mathbf{R}^{m} \times \mathbf{R}^{n}$ to $\mathfrak{q}$ by

$$
F(x, y)=e^{y_{1} b_{1}} \cdots e^{y_{n} b_{n}}\left(X_{0}+\sum_{i=1}^{m} x_{i} u_{i}\right) .
$$

Then $F(0,0)=X_{0}$ and $d F$ is non-singular at $(0,0)$.

This lemma follows from the definition.

Let $U_{0}$ and $V_{0}$ be open subsets of $\mathbf{R}^{m}$ and $\mathbf{R}^{n}$ containing the origins, such that $U_{0} \subset U^{\prime}$ and that $F \mid U_{0} \times V_{0}$ is an isomorphism. Put $\Omega_{0}=$ $F\left(U_{0} \times V_{0}\right)$. Then $\Omega_{0}$ is an open neighbourhood of $X_{0}$ and is in a one to one correspondence with $U_{0} \times V_{0}$. For simplicity, put $f^{\sim}(x, y)=f(F(x, y))$ for any $f \in \mathscr{A}\left(\Omega_{0}\right)$. Similarly, for any $D \in \mathscr{D}\left(\Omega_{0}\right)$, define a differential operator $D^{\sim}$ on $U_{0} \times V_{0}$ by $\left(D^{\sim} h\right)(x, y)=\left(D\left(h \circ F^{-1}\right)(F(x, y))\right)$. Since $F$ is bijective, $D^{\sim}$ is well-defined. Let $\widetilde{T}\left(U_{0} \times V_{0}\right)$ be the left ideal of $\mathscr{D}\left(U_{0} \times V_{0}\right)$ generated by the vector fields $\tau(X)^{\sim}(\forall X \in \mathfrak{h})$.

Lemma 3.7. $D_{y_{i}}(1 \leq i \leq n)$ are contained in $\widetilde{T}\left(U_{0} \times V_{0}\right)$.

Proof. Define analytic functions $f_{i j}(y)$ by $\operatorname{Ad}\left(e^{y_{1} b_{1}} \ldots e^{y_{i-1} b_{i-1}}\right) b_{i}=$ $\sum_{j=1}^{k} f_{i j}(y) X_{j}$, where $\left\{X_{1}, \cdots, X_{k}\right\}$ is a basis of $\mathfrak{h}(k=\operatorname{dim} \mathfrak{h})$. Then it follows that

$$
D_{y_{i}}=\sum_{j=1}^{k} f_{i j}(y) \tau\left(X_{j}\right)^{\sim} \in \widetilde{\mathscr{T}}\left(U_{0} \times V_{0}\right) . \quad \text { q.e.d. }
$$

Lemma 3.8. For each $i(1 \leq i \leq n)$, the local expression of $\tau\left(b_{i}\right)^{\sim}$ at $(x, 0)$ coincides with $-D_{y_{i}}$. 
Proof. Take $h \in \mathscr{A}\left(U_{0} \times V_{0}\right)$ and calculate $\left(\tau\left(b_{i}\right)^{\sim} h\right)(x, 0)$. For simplicity, put $f=h \circ F^{-1} \in \mathscr{A}\left(\Omega_{0}\right)$. Then

$$
\begin{aligned}
\left(\tau\left(b_{i}\right)^{\sim} h\right)(x, 0) & =\left(\tau\left(b_{i}\right) f\right)(F(x, 0)) \\
& =\left(\tau\left(b_{i}\right) f\right)\left(X_{0}+\sum_{j} x_{j} u_{j}\right) \\
& =\left.\frac{d}{d t} f\left(e^{-t b_{i}}\left(X_{0}+\sum_{j} x_{j} u_{j}\right)\right)\right|_{t=0 .}
\end{aligned}
$$

Since $e^{-t b_{i}}\left(X_{0}+\sum_{j} x_{j} u_{j}\right)=F(x, y)$ with $y_{k}=-\delta_{k i} t(1 \leq k \leq n)$, we find that

$$
\left.\frac{d}{d t} f\left(e^{-t b_{i}}\left(X_{0}+\sum_{j} x_{j} u_{j}\right)\right)\right|_{t=0}=-\left(D_{y_{i}} h\right)(x, 0) .
$$

Hence it follows that $\left(\tau\left(b_{i}\right)^{\sim} h\right)(x, 0)=-\left(D_{y_{i}} h\right)(x, 0)$. This holds for any $h \in \mathscr{A}\left(U_{0} \times V_{0}\right)$ and the lemma follows.

q.e.d.

Lemma 3.9. Take $X_{1}, \cdots, X_{k} \in \mathfrak{h}$ and $Y_{1}, \cdots, Y_{l} \in \mathfrak{q}$ and define the differential operator $P$ on $\mathfrak{q}$ by

$$
(P f)(Y)=\frac{\partial^{k}}{\partial t_{1} \cdots \partial t_{k}} \frac{\partial^{l}}{\partial s_{1} \cdots \partial s_{l}} f\left(\left.e^{t_{1} X_{1}} \cdots e^{t_{k} X_{k}}\left(Y+\sum_{j=1}^{l} s_{j} Y_{j}\right)\right|_{t=s=0}\right.
$$

for any $f \in \mathscr{A}(\mathfrak{q})$. On the other hand, define $g=X_{1} \cdots X_{k} \in U\left(\mathfrak{h}_{c}\right)$ and $p=$ $Y_{1} \cdots Y_{l} \in S\left(\mathfrak{q}_{c}\right)$. Then the local expression of $P$ at $Y \in \mathfrak{q}$ coincides with $\partial\left(\Gamma_{Y}(g \otimes p)\right)$.

This follows from the definitions of $\Gamma_{Y}(g \otimes p)$ and $P$.

(3.5) If $p \in S\left(\mathfrak{q}_{c}\right)$, then $\partial(p)$ is a differential operator on $\mathfrak{q}$ and also $\partial(p)^{\sim}$ is a differential operator on $U_{0} \times V_{0}$. Then it follows that

$$
\partial(p)^{\sim}=\sum_{\alpha \in \mathbb{N}^{m}} \sum_{\beta \in \mathbb{N}^{n}} a_{\alpha \beta}(x, y) D_{x}^{\alpha} D_{y}^{\beta},
$$

where each $a_{\alpha \beta}(x, y)$ is an analytic function on $U_{0} \times V_{0}$. On the other hand, let $\alpha_{u}(p)$ be the radial component of $p$ at $u \in U^{\prime}$. For simplicity, put $u^{\alpha}=u_{1}^{\alpha_{1}} \cdots u_{m}^{\alpha_{m}} \in S\left(U_{c}\right)\left(\alpha \in \mathbf{N}^{m}\right)$. Then, from Lemma 3.3, we can define analytic functions $f_{\alpha}(x)\left(\alpha \in \mathbf{N}^{m}\right)$ such that $\alpha_{u}(p)=\sum_{\alpha \in \mathbb{N}^{m}} f_{\alpha}(x) u^{\alpha}$ for any $u=\sum_{j=1}^{m} x_{j} u_{j} \in U^{\prime}$. Corresponding to this expression, we define the differential operator $Q\left(x, D_{x}\right)$ on $U^{\prime}\left(\subset \mathbf{R}^{m}\right)$ by

$$
Q\left(x, D_{x}\right)=\sum_{\alpha \in \mathbb{N}^{m}} f_{\alpha}(x) D_{x}^{\alpha} .
$$


Lemma 3.10. $Q$ coincides with $\sum_{\alpha \in \mathbb{N}^{m}} a_{\alpha 0}(x, 0) D_{x}^{\alpha}$.

Proof. It follows from Lemma 3.8 and 3.9 that for any $\alpha \in \mathbf{N}^{m}$ and $\beta \in \mathbf{N}^{n}, D_{x}^{\alpha} D_{y}^{\beta}$ coincides with the local expression of $\partial\left(\Gamma_{X_{0}+\Sigma x_{j} u_{j}}\left(b^{\beta} \otimes u^{\alpha}\right)\right)^{\sim}$ at $(x, 0)$, where $b^{\beta}$ denotes $b_{1}^{\beta_{1}} \cdots b_{n}^{\beta_{n}}$ for all $\beta \in \mathbf{N}^{n}$. Hence the local expression of $\partial(p)^{\sim}$ at $(x, 0) \in U_{0} \times V_{0}$ coincides with that of

$$
\sum_{\alpha \in \mathbb{N}^{m}} \sum_{\beta \in \mathbb{N}^{n}} a_{\alpha \beta}(x, 0) \partial\left(\Gamma_{X_{0}+\Sigma x_{j} u_{j}}\left(b^{\beta} \otimes u^{\alpha}\right)\right)^{\sim} .
$$

On the other hand, from Lemma 3.3, we can choose $g_{j} \in \mathbb{S}_{+}\left(V_{c}\right), p_{j} \in$ $S\left(U_{c}\right)$, polynomials $q_{j}(u)$ on $U(1 \leq i \leq N)$ and an integer $r \geq 0$ such that

$$
p=\alpha_{u}(p)+\zeta(u)^{-r} \sum_{j=1}^{N} q_{j}(u) \Gamma_{X_{0}+u}\left(g_{j} \otimes p_{j}\right)
$$

for $u \in U^{\prime}$. Put $\zeta^{\sim}(x)=\zeta(u), q_{j}^{\sim}(x)=q_{j}(u)$ for $u=\sum_{i} x_{i} u_{i}$. Then we find that the local expression of $\partial(p)^{\sim}$ at $(x, 0)$ coincides with that of

$$
Q+\zeta^{\sim}(x)^{-r} \sum_{j=1}^{N} q_{j}^{\sim}(x) \partial\left(\Gamma_{X_{0}+\Sigma x_{j} u_{j}}\left(g_{j} \otimes p_{j}\right)\right)^{\sim} .
$$

Comparing (3.5.3) and (3.5.5) and noting (3.5.2), we obtain the lemma.

q.e.d.

For any $p \in S\left(\mathfrak{q}_{c}\right)$, we denote by $\Delta\left(\partial(p)^{\sim}\right)$ the differential operator $Q\left(x, D_{x}\right)$ defined in (3.5.2) and call it the radial component of $\partial(p)^{\sim}$ on $U_{0}$. By definition, $\Delta\left(\partial(p)^{\sim}\right)$ coincides with the differential operator $\partial\left(\alpha_{u}(p)\right)$ on $U_{0}$ by the correspondence $\left(x_{1}, \cdots, x_{m}\right) \rightarrow \sum_{j} x_{j} u_{j}$. In this sense, $\Delta\left(\partial(p)^{\sim}\right)$ is nothing but $\Delta(\partial(p))$ defined in (3.3). Hence we confuse these ones in the sequel.

(3.6) Let $u$ be a locally invariant hyperfunction defined on the open subset $\Omega_{0}$ of $\mathfrak{q}$ and let $p$ be an element of $S\left(\mathfrak{q}_{c}\right)^{H}$. The next lemma is a direct consequence of Lemmas 3.7 and 2.1.

Lemma 3.11. The hyperfunction $\tilde{u^{\sim}}(x, y)$ on $U_{0} \times V_{0}$ is constant with respect to the variable $y$.

This combined with the assumption on $p$ implies that $\tilde{u^{\sim}}(x, y)$ and $(\partial(p) u)^{\sim}(x, y)$ can be restricted on the closed subset $U_{0} \simeq U_{0} \times\{0\} \subset U_{0} \times V_{0}$. Then the radial component $\Delta\left(\partial(p)^{\sim}\right)$ has the following meaning.

Theorem 3.12. Let $u$ and $p$ be as above. Put $v(x)=u^{\sim}(x, 0)$, the restriction of $u$ to $U_{0}$. Then

$$
\left(\partial(p)^{\sim} u\right)(x, 0)=\Delta\left(\partial(p)^{\sim}\right) v(x) .
$$


This follows from the definition of $\Delta\left(\partial(p)^{\sim}\right)$ and Lemmas 3.7-3.10 (cf. [HC2, Lemma 10]).

\section{§ 4. The radial component of the pseudo-Laplacian on $\mathfrak{q}$}

(4.1) Retain the notation in the previous sections. Let $\omega$ be the Casimir polynomial on $\mathfrak{q}$.

Definition 4.1. The differential operator $\partial(\omega)$ is called the pseudoLaplacian on $\mathfrak{q}$.

The purpose of this section is to calculate the radial components of $\partial(\omega)$ for certain elements of $\mathfrak{q}$.

(4.2) Let $A_{0}$ be a semisimple element of q. Similarly as in (1.5), define $z_{\partial}, z^{ \pm}, z_{s}, z_{s}^{ \pm}, \mathfrak{c}^{-}$. Take a Cartan subspace $\mathfrak{a}$ of $\mathfrak{q}$ containing both $A_{0}$ and $\mathfrak{c}^{-}$and let $\Sigma=\Sigma(\mathfrak{a})$ be the root system of $\left(\mathfrak{g}_{c}, \mathfrak{a}_{c}\right)$ (cf. (1.4)). For any root $\lambda \in \Sigma$, choose a $\mathbf{C}$-basis $X_{\lambda, 1}, \cdots, X_{\lambda, m_{\lambda}}$ of $\mathrm{g}_{c}^{\lambda}\left(m_{\lambda}=\operatorname{dim}_{\mathrm{c}} \mathrm{g}_{c}^{\lambda}\right)$ such that $B\left(X_{\lambda, i}, \sigma X_{\lambda, j}\right)=-\delta_{i j}\left(i, j=1, \cdots, m_{\lambda}\right)$. Put $\mathfrak{a}^{-}=\mathfrak{a} \cap z_{s}^{-}$. Then $\mathfrak{a}=$ $\mathfrak{a}^{-}+\mathfrak{c}^{-}$, and $\mathfrak{a}^{-}$and $\mathfrak{c}^{-}$are orthogonal with respect to the Killing form. Let $A_{1}, \cdots, A_{p}$ be a $\mathbf{C}$-basis of $a_{c}^{-}$such that $B\left(A_{i}, A_{j}\right)=\delta_{i j}$ and let $C_{1}, \cdots, C_{q}$ be a $\mathbf{C}$-basis of $\mathfrak{c}_{c}^{-}$such that $B\left(C_{i}, C_{j}\right)=\delta_{i j}$. As usual, identify $\mathfrak{q}_{c}$ with its dual by the Killing form. Then it follows from the definition that

$$
\omega=\sum_{i=1}^{p} A_{i}^{2}+\sum_{i=1}^{q} C_{i}^{2}+\frac{1}{2} \sum_{\lambda \in \Sigma^{+}} \sum_{j=1}^{m_{\lambda}}\left(X_{\lambda, i}-\sigma X_{\lambda, i}\right)^{2} .
$$

(Here $\Sigma^{+}$is the positive system of $\Sigma$ for an order fixed hereafter.) Define

$$
\begin{aligned}
& \Sigma_{0}=\left\{\lambda \in \Sigma ; \lambda\left(A_{0}\right)=0\right\} \\
& \Sigma_{1}=\left\{\lambda \in \Sigma ; \lambda\left(A_{0}\right) \neq 0\right\} \\
& \Sigma_{j}^{+}=\Sigma^{+} \cap \Sigma_{j} \quad(j=0,1) .
\end{aligned}
$$

Using these, we also define

$$
\begin{aligned}
& V_{c}^{ \pm}=\sum_{\lambda \in \Sigma_{1}^{+}} \sum_{j=1}^{m_{\lambda}} \mathbf{C}\left(X_{\lambda, i} \pm \sigma X_{\lambda, i}\right) \\
& V^{+}=V_{c}^{+} \cap \mathfrak{h}, \quad V^{-}=V_{c}^{-} \cap \mathfrak{q} .
\end{aligned}
$$

Then it is clear that $\mathfrak{h}=\mathfrak{z}^{+}+V^{+}$and $\mathfrak{q}=z^{-}+V^{-}$are direct sums. Moreover, we have $\left[A_{0}, \mathfrak{h}\right]=\left[A_{0}, V^{+}\right]=V^{-}$and $\operatorname{dim} V^{+}=\operatorname{dim} V^{-}$. Defining the linear mapping $\eta_{u}$ of $z^{-} \times V^{+}$to $\mathfrak{q}$ by $\eta_{u}(w, v)=w+[u, v]$, we find that $\eta_{A_{0}}$ is bijective. Hence $\xi(u)=\operatorname{det}\left(\eta_{u} \circ \eta_{A_{0}}^{-1}\right)\left(u \in z^{-}\right)$is well-defined. The next 
lemma follows from the definition.

Lemma 4.2. $\xi(A)=\prod_{\lambda \in \Sigma_{1}^{+}}\left[\lambda(A) / \lambda\left(A_{0}\right)\right]^{m_{\lambda}}$ for all $A \in \mathfrak{a}$.

Put $\left(z^{-}\right)^{\prime}=\left\{u \in z^{-} ; \xi(u) \neq 0\right\}$. Then the following statements are consequences of the results in (3.2).

Lemma 4.3. For any $Z \in\left(z^{-}\right)^{\prime}, \Gamma_{z}$ defines a bijective mapping of $\widehat{S}\left(V_{c}^{+}\right) \otimes S\left(\jmath_{c}^{-}\right)$to $S\left(\mathfrak{q}_{c}\right)$.

Corollary 1. Fix $p \in S\left(\mathfrak{q}_{c}\right)$. Then for any $Z \in\left(\mathfrak{z}^{-}\right)^{\prime}$, there exist unique elements $\alpha_{z}(p) \in S\left(\partial_{c}^{-}\right)$and $\beta_{z}(p) \in \mathbb{S}_{+}\left(V_{c}^{+}\right) \otimes S\left(\partial_{c}^{-}\right)$such that $p-\alpha_{z}(p)=$ $\Gamma_{z}\left(\beta_{z}(p)\right)$. Moreover $d^{0} \alpha_{z}(p) \leq d^{0} p$.

Corollary 2. For any $p \in S\left(\mathfrak{q}_{c}\right)$, we can choose an integer $r \geq 0$ such that the mappings $Z \rightarrow \xi(Z)^{r} \alpha_{Z}(p)$ and $Z \rightarrow \xi(Z)^{r} \beta_{Z}(p)\left(Z \in\left(\partial_{c}^{-}\right)^{\prime}\right)$ can be extended to polynomial mappings of $z_{c}^{-}$into $S\left(z_{c}^{-}\right)$and $\widetilde{S}\left(V_{c}^{+}\right) \otimes S\left(z_{c}^{-}\right)$, respectively.

Let $\omega_{s}$ (resp. $\omega_{c}$ ) be the restriction of $\omega$ to $z_{s}^{-}\left(\right.$resp. $\left.c^{-}\right)$. Then $\omega_{s}$ (resp. $\omega_{c}$ ) is a non-degenerate quadratic form on ${ }_{\delta}^{-}$(resp. $\mathcal{c}^{-}$). Using $\omega_{s}$ and $\omega_{c}$, identify $\jmath_{s}^{-}$with its dual and $c^{-}$with its dual. Then it follows that

$$
\begin{aligned}
& \omega_{s}=\sum_{i=1}^{p} A_{i}^{2}+\frac{1}{2} \sum_{\lambda \in \Sigma_{0}^{+}} \sum_{j=1}^{m_{\lambda}}\left(X_{\lambda, i}-\sigma X_{\lambda, i}\right)^{2} \\
& \omega_{c}=\sum_{i=1}^{q} C_{i}^{2} .
\end{aligned}
$$

Lemma 4.4. Let $\Delta(\partial(\omega))$ be the differential operator on $z^{-}$obtained from $\partial(\omega)$ as we did in (3.5). Then

$$
\Delta(\partial(\omega))=\xi(u)^{-1 / 2}\left(\partial\left(\omega_{c}\right)+\partial\left(\omega_{s}\right)\right) \circ \xi(u)^{1 / 2}-\mu(u),
$$

where $\mu(u)=\xi(u)^{-1 / 2}\left(\partial\left(\omega_{c}\right)+\partial\left(\omega_{s}\right)\right) \xi(u)^{1 / 2}$ is an analytic function on $\left(z^{-}\right)^{\prime}$.

This lemma is shown by an argument similar to Lemma 18 in [HC3]. In the proof, we use the expression of $\omega$ in (4.2.1) and those of $\omega_{s}, \omega_{c}$ in (4.2.3). Note that in our case, the function $\mu(u)$ does not vanish in general.

Let $\mathrm{H}^{+}$and $H_{s}^{+}$be the analytic subgroups of $H$ corresponding to $z^{+}$ and $\jmath_{s}^{+}$, respectively. Then it follows from the definition that both $\xi(u)$ and $\mu(u)$ are $H^{+}$-invariant.

(4.3) Take a nilpotent element $X_{0}$ of $z_{s}^{-}$and let $\left(B_{0}, X_{0}, Y_{0}\right)$ be a normal $S$-triple for the pair $\left(\partial_{s}, \partial_{s}^{+}\right)$. In particular, $B_{0} \in \partial_{s}^{+}$and $Y_{0} \in \partial_{s}^{-}$. Then we have the next lemma which is shown in the way similar to the 
proof of Lemma 1.12. Though $\omega_{s}$ is not the Casimir polynomial on $z_{s}^{-}$, we only use in the proof that $\omega_{s}$ is non-degenerate.

Lemma 4.5. The following conditions are equivalent.

(i) $X_{0}$ is $\gamma_{s}^{-}$-distinguished.

(ii) $\omega_{s}(X)=0$ for all $X \in\left(\partial_{s}^{-}\right)_{X_{0}}$.

(iii) $\omega_{s}(X)=0$ for all $X \in\left(\partial_{s}^{-}\right)_{Y_{0}}$.

(iv) $\left(z_{s}^{-}\right)_{X_{0}} \cap\left(\bar{z}_{s}^{-}\right)_{Y_{0}}=0$.

It follows from Lemma 1.9 that there exists a Cartan involution $\theta$ on $z_{s}$ commuting with $\sigma$ such that $\theta:\left(B_{0}, X_{0}, Y_{0}\right) \rightarrow\left(-B_{0},-Y_{0},-X_{0}\right)$. Then $-B(X, \theta(Y))\left(X, Y \in \partial_{s}\right)$ is a positive definite bilinear form on $\partial_{s}$. This defines a Euclidean structure on $z_{s}$. The adjoint of $\operatorname{ad}_{3 s} X\left(X \in z_{s}\right)$ with respect to this structure is given by $-\left.\left(\operatorname{ad}_{g} \theta X\right)\right|_{z_{s}}$. Put $U=\left(z_{s}^{-}\right)_{Y_{0}}$ and choose an orthonormal basis $u_{1}, \cdots, u_{m}$ of $U$ such that $\left[B_{0}, u_{i}\right]=-\lambda_{i} u_{i}$ $(1 \leq i \leq m)(m=\operatorname{dim} U)$. Then each $\lambda_{i}$ is a non-negative integer. These follow from the arguments in $[\mathrm{vD}, \S 1]$ (cf. $\S 1)$. Since $Y_{0}$ is contained in $\left(\partial_{s}^{-}\right)_{Y_{0}}=U$, we may assume that $u_{1}=c Y_{0}\left(c=1 /\left\|Y_{0}\right\|\right)$. So $\lambda_{1}=2$. Let $x_{1}, \cdots, x_{m}$ be the coordinates with respect to this basis of $U$.

For any $u \in U$, similarly as in (3.2), we define the linear mapping $\psi_{u}$ of $U \times\left[z_{s}^{-}, Y_{0}\right]$ to $q$ by $\psi_{u}(w, v)=w+\left[X_{0}+u, v\right]$. Since $\gamma_{s}^{+}=\left[z_{s}^{-}, Y_{0}\right]+\left(\partial_{s}^{+}\right)_{X_{0}}$ is a direct sum, it follows that $\psi_{0}$ is bijective. Noting this, we put $\kappa(u)=$ $\operatorname{det}\left(\psi_{u} \circ \psi_{0}^{-1}\right)(u \in U)$ and $U^{\prime}=\{u \in U ; \kappa(u) \neq 0\}$ as in (3.2). Let $b_{1}, \cdots, b_{n}$ be an arbitrary basis of $\left[\tilde{o}_{s}^{-}, Y_{0}\right]$ and let $y_{1}, \cdots, y_{n}$ be the coordinates with respect to this basis on $\left[z_{s}^{-}, Y_{0}\right]$. Using the coordinates $y$ on $\left[z_{s}^{-}, Y_{0}\right]$ and $x$ on $U$, define the mapping $\Psi$ of $\mathbf{R}^{m} \times \mathbf{R}^{n}$ to $z_{s}^{-}$by the same way as (3.4.1):

$$
\Psi(x, y)=e^{y_{1} b_{1}} \cdots e^{y_{n} b_{n}}\left(X_{0}+\sum_{j=1}^{m} x_{j} u_{j}\right) .
$$

Then it follow from Lemma 3.6 that $\Psi(0,0)=X_{0}$ and $d \Psi$ is non-singular at $(0,0)$. Hence we define $U_{0}, V_{0}$ and $\Omega_{0}$ as in (3.2), in particular, $0 \in U_{0}$ $\subset \mathbf{R}^{m}, 0 \in V_{0} \subset \mathbf{R}^{n}$ and $\Psi \mid U_{0} \times V_{0}$ is a diffeomorphism and $\Omega_{0}=F\left(U_{0} \times V_{0}\right)$. Then $\Omega_{0}$ is an open neighbourhood of $X_{0}$ in $z_{s}^{-}$and $(x, y)$ is regarded as a coordinate system on $\Omega_{0}$.

Since $\omega_{s}$ is contained in $S\left(\partial_{s}^{-}\right)$, we can define the radial component

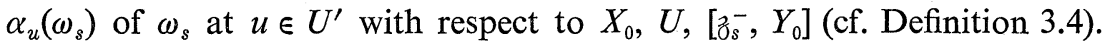
Let $\Delta\left(\partial\left(\omega_{s}\right)\right)$ be the differential operator on $U^{\prime}$ such that $\Delta_{u}\left(\partial\left(\omega_{s}\right)\right)=$ $\partial\left(\alpha_{u}\left(\omega_{s}\right)\right)$ for all $u \in U^{\prime} .\left(\Delta_{u}\left(\partial\left(\omega_{s}\right)\right)\right.$ denotes the local expression of $\partial\left(\omega_{s}\right)$ at $u$. $)$

Lemma 4.6. The homogeneous part of degree 2 of $\Delta_{0}\left(\partial\left(\omega_{s}\right)\right)$ is zero if and only if $X_{0}$ is ${ }_{z-}^{-}$-distinguished.

This is shown by an argument similar to Lemma 33 in [HC2] (cf. [vD, Lemma 13]). 
Theorem 4.7. Assume that $X_{0}$ is $z_{s}^{-}$-distinguished. Then there exist analytic functions $a_{i j}(x)$ and $a_{i}(x)$ on $U_{0}$ satisfying $a_{i j}(0)=0(1<i, j \leq m)$, such that

$$
\begin{aligned}
\left\|X_{0}\right\| \Delta\left(\partial\left(\omega_{s}\right)\right)= & 2 x_{1} \frac{\partial^{2}}{\partial x_{1}^{2}}+\left(\operatorname{dim} z_{s}^{-}\right) \frac{\partial}{\partial x_{1}}+\sum_{i=2}^{m}\left(\lambda_{i}+2\right) x_{i} \frac{\partial^{2}}{\partial x_{1} \partial x_{i}} \\
& +\sum_{1<i \leq j \leq m} a_{i j}(x) \frac{\partial^{2}}{\partial x_{i} \partial x_{j}}+\sum_{i=2}^{m} a_{i}(x) \frac{\partial}{\partial x_{i}} .
\end{aligned}
$$

This is shown by an argument similar to Theorem 14 in [vD]. It should be noted that though $\omega_{s}$ is not the Casimir polynomial on $\gamma_{s}^{-}$, we only used in the proof that $\omega_{s}$ is non-degenerate.

Remark 4.8. A prototype of the formula in Theorem 4.7 seems to have first appeared in Atiyah's lecture note [A] as a literature. T. Kawazoe and the author obtained the formula (4.3.2) a few years ago being suggested by the note [A]. Afterwards, T. Oshima informed the author of van Dijk's article [vD] in which the formula was also shown. Our method was based on direct calculations by using the expression in (4.2.1). On the other hand, van Dijk's method is on the Euler operator and is therefore very simple and elegant compared with ours.

Last we comment on the differential operator $\partial\left(\omega_{c}\right)$. Since $\omega_{c}$ is a quadratic form on the vector space $c^{-}$, with respect to an appropriate basis $c_{1}, \cdots, c_{q}$ of $\mathfrak{c}^{-}\left(q=\operatorname{dim} \mathrm{c}^{-}\right)$, we have

$$
\partial\left(\omega_{c}\right)=D_{t_{1}}^{2}+\cdots+D_{t_{q_{1}}}^{2}-D_{t_{q_{1}+1}}^{2}-\cdots-D_{t_{q}}^{2},
$$

where $t_{1}, \cdots, t_{q}$ are the coordinates with respect to the basis and $q_{1}$ is a certain integer such that $q_{1} \leq q$.

\section{§5. Invariant spherical hyperfunctions on $\mathfrak{q}$}

(5.1) For an arbitrary linear form $\Lambda$ on $\mathfrak{a}_{c}$, we define a system of differential equations $\mathscr{M}_{A}$ on $\mathfrak{q}$ defined by

$$
\mathscr{M}_{A}\left\{\begin{array}{l}
\partial(P) u=P(\Lambda) u \quad \text { for all } P \in S\left(\mathfrak{q}_{c}\right)^{H} \\
\tau(Y) u=0 \quad \text { for all } Y \in \mathfrak{h} .
\end{array}\right.
$$

It is provable that $\mathscr{M}_{A}$ is holonomic in the sense of Sato-Kashiwara.

Definition 5.1. A hyperfunction solution $u$ to the system $\mathscr{M}_{A}$ is called an invariant spherical hyperfunction $(=\mathrm{ISH})$ on $q$ with the infinitesimal character $\Lambda$. Moreover, an ISH $u$ is called singular if $\operatorname{Supp}(u)$ is contained 
in the set $\mathfrak{q}-\mathfrak{q}^{\prime}$.

First we give a remark on the relation between ISH's and invariant eigendistributions ( $=$ IED) on a semisimiple Lie algebra. Let $g_{0}$ be a real semisimple Lie algebra and put $\mathfrak{g}=\mathfrak{g}_{0} \oplus \mathfrak{g}_{0}$. Define the involution $\sigma$ on $\mathfrak{g}$ by $\sigma(X, Y)=(Y, X)\left(X, Y \in g_{0}\right)$. Then we obtain the direct sum decomposition $\mathfrak{g}=\mathfrak{h}+\mathfrak{q}$ for $\sigma$. In this case, $\mathfrak{h}=\left\{(X, X) ; X \in \mathfrak{g}_{0}\right\}$ and $\mathfrak{q}=\{(X,-X)$; $\left.X \in \mathfrak{g}_{0}\right\}$. Hence $\mathfrak{h} \simeq \mathfrak{q} \simeq \mathfrak{g}_{0}$. Put $G_{0}=\operatorname{Int}\left(\mathfrak{g}_{0}\right)$ and $G=\operatorname{Int}(\mathfrak{g})$. Then $G=$ $G_{0} \times G_{0}$ and $H$ is equal to the diagonal subgroup $\left\{(g, g) ; g \in G_{0}\right\}$ of $G$. Moreover the action of $H$ on $\mathfrak{q}$ is identified with the adjoint action of $G_{0}$ on $\mathfrak{g}_{0}$. Under the identification, an ISH on $\mathfrak{q}$ is nothing but an IED on $\mathfrak{g}_{0}$.

As to a singular IED on a semisimple Lie algebra, Harish-Chandra obtained the following result which plays a fundamental role in the proof of his famous theorem that any IED on a real semisimple Lie algebra is $L^{1}$-local (cf. [HC1]).

Theorem $\mathrm{HC}([\mathrm{HC} 3])$. There exists no non-zero singular invariant eigendistribution on a real semisimple Lie algebra.

As will be seen in Section 6, there exists a non-zero singular ISH on $\mathfrak{q}$ for some symmetric pair $(\mathfrak{g}, \mathfrak{h})$ and therefore an analogue of Theorem HC to the case of the tangent space of a symmetric space does not hold in general. But we can prove an analogue for symmetric pairs which satisfy some additional condition.

(5.2) The main result of this paper is the following theorem which is an analogue of Theorem $\mathrm{HC}$.

Theorem 5.2. Let $(\mathfrak{g}, \mathfrak{h})$ be a symmetric pair. If $\delta_{\mathfrak{q}}(Z)>0$ holds for any $\mathfrak{q}$-distinguished element $Z$ of $\mathfrak{q}$, then there exists no non-zero singular invariant spherical hyperfunction on $\mathfrak{q}$.

Remark. We shall discuss the condition of the theorem in Section 6.

Proof. Let $u$ be a singular ISH on $\mathfrak{q}$. Then, by definition, Supp $(u)$ is contained in $\mathfrak{q}-\mathfrak{q}^{\prime}$. Assuming that $u \neq 0$, we lead a contradiction. Hence assume that $u \neq 0$ and therefore that $\operatorname{Supp}(u) \neq \emptyset$. Take $Z_{0} \in \operatorname{Supp}(u)$. Let $Z_{0}=A_{0}+X_{0}$ be its Jordan decomposition, where $A_{0} \in \mathscr{S}(\mathfrak{q})$ and $X_{0} \in$ $\mathscr{N}(\mathfrak{q})$. As in (1.5), define $z^{ \pm}, z^{ \pm}, \mathfrak{c}^{-}$for the semisimple element $A_{0}$. Now $S_{k}$ is the set of those $Z_{0}$ such that rank $z_{s}^{-}=k$. Then $\operatorname{Supp}(u)=\bigcup_{k=0}^{l} S_{k}$, where $l=\operatorname{rank} \mathfrak{q}$. Since $\operatorname{Supp}(u) \subset \mathfrak{q}-\mathfrak{q}^{\prime}$, it follows that $A_{0}$ is not $\mathfrak{q}$-regular. This implies that $S_{0}=\emptyset$.

Now assume that $S_{1}=\cdots=S_{k-1}=\emptyset$ and $S_{k} \neq \emptyset$. Then we may assume from the first that $Z_{0}$ is contained in $S_{k}$. Retain the notation 
above. Let $H_{s}^{+}$be the analytic subgroup of $H$ corresponding to $z_{s}^{+}$. Then $H_{s}^{+}$acts on $z_{s}^{-}$. Since $\mathscr{N}\left(\partial_{s}^{-}\right)$is decomposed into finitely many $H_{s}^{+}$-orbits (cf. Lemma 1.5), we can write $\mathscr{N}\left(\partial_{s}^{-}\right)=O_{1} \cup \cdots \cup O_{\nu}$, where the $O_{j}$ are disjoint $H_{s}^{+}$-orbits and for $1 \leq j \leq \nu, \mathscr{N}_{j}=O_{j} \cup \cdots \cup O_{\nu}$ is a closed set containing $O_{j}$ as an open subset. Since $Z_{0}=A_{0}+X_{0}$ is contained in Supp $(u)$ and also in $A_{0}+\mathscr{N}\left(\overline{o s}_{s}^{-}\right)$, it follows that $\operatorname{Supp}(u) \cap\left(A_{0}+\mathscr{N}\left(z_{s}^{-}\right)\right)$ $\neq \emptyset$. Noting this, we may assume that $\operatorname{Supp}(u) \cap\left(A_{0}+O_{i}\right)=\emptyset$ for $i=$ $1, \cdots, j-1$ and $\operatorname{Supp}(u) \cap\left(A_{0}+O_{j}\right) \neq \emptyset$ for some $j$. It is clear that $\operatorname{Supp}(u) \cap\left(A_{0}+O_{j}\right)$ is contained in $S_{k}$. Hence we may assume from the first that $X_{0}$ is contained in $O_{j}$.

For the element $A_{0}$, we define $V^{+}$as in (4.2.2). Let $f_{1}, \cdots, f_{d}$ be a basis of $V^{+}$and let $z_{1}, \cdots, z_{d}$ be the coordinates with respect to this basis of $V^{+}$. Similarly, we retain the notation in (4.3). So let $\left(B_{0}, X_{0}, Y_{0}\right)$ be a normal $S$-triple. Then we define $U=\left(\partial_{s}^{-}\right)_{Y_{0}}$. Take the basis $u_{1}, \cdots, u_{m}$ of $U$ and its coordinates $x_{1}, \cdots, x_{m}$ as in (4.3). Moreover let $b_{1}, \cdots, b_{n}$ be the basis of $\left[z_{s}^{-}, Y_{0}\right]$ and $y_{1}, \cdots, y_{n}$ its coordinates. Last let $c_{1}, \cdots, c_{q}$ be the basis of $\mathrm{c}^{-}$and $t_{1}, \cdots, t_{q}$ its coordinates. Using these coordinates, we define the map $\Phi$ of $\mathbf{R}^{q} \times \mathbf{R}^{m} \times \mathbf{R}^{n} \times \mathbf{R}^{d}$ to $q$ by

$$
\begin{aligned}
& \Phi\left(t_{1}, \cdots, t_{q}, x_{1}, \cdots, x_{m}, y_{1}, \cdots, y_{n}, z_{1}, \cdots, z_{d}\right) \\
= & e^{z_{1} f_{1}} \cdots e^{z_{d f d}}\left(A_{0}+\sum_{i=1}^{q} t_{i} c_{i}+e^{y_{1} b_{1}} \cdots e^{y_{n} b_{n}}\left(X_{0}+\sum_{\alpha=1}^{m} x_{\alpha} u_{\alpha}\right)\right) \\
= & e^{z_{1} f_{1}} \cdots e^{z_{d f d}}\left(A_{0}+\sum t_{i} c_{i}+\Psi(x, y)\right),
\end{aligned}
$$

where $\Psi(x, y)$ is the mapping defined in (4.3.1). Then it follows that $\Phi(0)$ $=A_{0}+X_{0}$ and $\Phi$ induces a diffeomorphism between an open neighbourhood of the origin in $\mathbf{R}^{q+m+n+d}$ and that of $Z_{0}$ in $\mathfrak{q}$. Hence take open subsets $T_{0}, U_{0}, V_{0}$ and $W_{0}$ of $\mathbf{R}^{q}, \mathbf{R}^{m}, \mathbf{R}^{n}$ and $\mathbf{R}^{d}$ containing the origins, respectively, such that $\Phi \mid T_{0} \times U_{0} \times V_{0} \times W_{0}$ is bijective. Put $\Xi=\Phi\left(T_{0} \times U_{0} \times V_{0} \times W_{0}\right)$ and the following identifications are used in the subsequent discussion:

$$
\begin{aligned}
T_{0} & \simeq T_{0} \times\{0\} \subset T_{0} \times U_{0} \simeq T_{0} \times U_{0} \times\{0\} \subset T_{0} \times U_{0} \times V_{0} \\
& \simeq T_{0} \times U_{0} \times V_{0} \times\{0\} \subset T_{0} \times U_{0} \times V_{0} \times W_{0} .
\end{aligned}
$$

Replacing $U_{0}$ and $V_{0}$ with small ones if necessary, we can show by an argument similar to Lemma 22 in [V] (cf. [vD, Lemma 17]) that $\mathscr{N}_{j} \cap\{\Phi(t, x$, $y, z) \in \Xi ; t=z=0\} \subseteq O_{j}$.

Since $u$ is locally invariant, it follows from Lemma 3.11 that $\tilde{u}(t, x, y, z)=u(\Phi(t, x, y, z))$ which is a hyperfunction on $T_{0} \times U_{0} \times V_{0} \times W_{0}$ is constant with respect to the variables $z_{1}, \cdots, z_{d}$. So put $u_{1}(t, x, y)=$ $\left.\tilde{u}(t, x, y, z)\right|_{z=0}$. This is a well-defined hyperfunction on the open subset $T_{0} \times U_{0} \times V_{0}$ of $\mathbf{R}^{q+m+n}$ containing the origin $0=\Phi^{-1}\left(Z_{0}\right)$. Moreover 
$\tilde{u}(t, x, y, z)=u_{1}(t, x, y)$ holds on $T_{0} \times U_{0} \times V_{0} \times W_{0} . \quad$ On the other hand, since $u$ is an ISH, we have

$$
(\partial(\omega)-\omega(\Lambda)) u=0
$$

for a linear form $\Lambda$ on $\mathfrak{a}_{c}$ which is the complexification of a Cartan subspace $\mathfrak{a}$ of $\mathfrak{q}$. Let $\xi$ be the function on $z^{-}$defined for $A_{0}$ as in (4.2). Replacing $T_{0}, U_{0}, V_{0}$ with small ones if necessary, we may assume from the first that $\xi \neq 0$ on $\Phi\left(T_{0} \times U_{0} \times V_{0}\right)$. So there exists an analytic function $\rho(t, x, y)$ on $T_{0} \times U_{0} \times V_{0}$ such that $\xi(\Phi(t, x, y, 0))=\rho(t, x, y)^{2}$ holds on $T_{0} \times$ $U_{0} \times V_{0}$. Put $u_{2}(t, x, y)=\rho(t, x, y)^{-1} u_{1}(t, x, y)$. Then it follows from Lemma 4.4 that

$$
\left\{\partial\left(\omega_{c}\right)^{\sim}+\partial\left(\omega_{s}\right)^{\sim}-\mu^{\sim}(t, x, y)-\omega(\Lambda)\right\} u_{2}=0 .
$$

where $\mu^{\sim}=\mu \circ \Phi$ (cf. Lemma 4.4). Since $\rho \circ \Phi^{-1}$ is $H_{s}^{+}$-invariant, it follows that

$$
\tau(Y)^{\sim} u_{2}=0 \quad \text { for all } Y \in z^{+},
$$

where the vector field $\tau(Y)^{\sim}$ is defined similarly as we did in (3.4). Since $z^{+}$commutes with $\mathfrak{c}^{-}$, we find from Lemma 3.11 and (5.2.2) that

$$
\frac{\partial}{\partial y_{i}} u_{2}(t, x, y)=0 \quad(i=1, \cdots, n) .
$$

Hence $u_{2}(t, x, y)$ is constant with respect to $y$. Noting this, we put $u_{3}(t, x)$ $=\left.u_{2}(t, x, y)\right|_{y=0}$. Then $u_{3}(t, x)$ is a well-defined hyperfunction on the open subset $T_{0} \times U_{0}$ of $\mathbf{R}^{q+m}$ containing $0=\Phi^{-1}\left(Z_{0}\right)$. The assumption combined with Lemma 17 (iii) in [vD] implies that Supp $\left(u_{3}\right)$ is contained in the set $T_{0}$. On the other hand, $u_{3}$ satisfies the differential equation

$$
\left(\Delta\left(\partial\left(\omega_{s}\right)\right)+\partial\left(\omega_{c}\right)-\mu^{\sim}(t, x, 0)-\omega(\Lambda)\right) u_{3}=0 .
$$

We can show under the assumption of the theorem that there exists no hyperfunction $u_{3}(y, t)$ satisfying the differential equation (5.2.3) and that Supp $\left(u_{3}\right) \subset T_{0}$. In fact, if $Z_{0}$ is not $q$-distinguished, that is, if $X_{0}$ is not $\partial_{s}^{-}$-distinguished nilpotent, it follows from (5.2.3), Lemma 4.6 and Proposition 2.2 that $u_{3}=0$. On the other hand, if $X$ is $z_{s}^{-}$-distinguished nilpotent, by means of the formula (4.3.2) for $\Delta\left(\partial\left(\omega_{s}\right)\right)$ and the assumption $\delta_{z \bar{s}}\left(X_{0}\right)>0$, we can conclude from Proposition 2.5 that $u_{3}=0$. Note that the condition $\delta_{z \bar{s}}\left(X_{0}\right)>0$ implies the condition (2.3.20) of Proposition 2.5. So we find that $u_{3}=0$ on $T_{0} \times U_{0}$, in other words, $u=0$ in the neighbourhood $\Xi$ of $Z_{0}$ in $\mathfrak{q}$. This contradicts the assumption that $Z_{0} \in \operatorname{Supp}(u)$. We have thus 
shown the theorem completely.

This theorem is restated in a slightly familiar form.

Theorem 5.3. Retain the assumption in Theorem 5.2 for a symmetric pair $(\mathfrak{g}, \mathfrak{h})$. Let $u(X)$ be an invariant spherical hyperfunction on $\mathfrak{q}$. Then one has:

(i) The restriction $u \mid \mathfrak{q}^{\prime}$ to $\mathfrak{q}^{\prime}$ is real analytic.

(ii) If $u \mid \mathfrak{q}^{\prime}=0$, then $u=0$ on the whole space $\mathfrak{q}$.

Proof. (ii) is a direct consequence of Theorem 5.2. On the other hand, (i) is proved if one can show the existence of an elliptic operator $P$ defined in a neighbourhood of an arbitrary element of $q^{\prime}$ such that $P u=0$. We are going to show this along the line of [A]. Hence let $X_{0}$ be an arbitrary element of $q^{\prime}$. Then there exists a Cartan subspace $a$ containing $X_{0}$. Clearly $\mathfrak{a}$ is uniquely determined by $X_{0}$ and $X_{0}$ is contained in $\mathfrak{a}^{\prime}$ (cf. (1.4)). Take a basis $\left\{h_{1}, \cdots, h_{l}\right\}$ of $\mathfrak{a}$. Then $Z=\sum_{i=1}^{l} h_{i}^{2}$ is contained in $S\left(\mathfrak{a}_{c}\right)$. If $W$ is the Weyl group of $\sum(\mathfrak{a})$ which is the root system of $\left(\mathfrak{g}_{c}, \mathfrak{a}_{c}\right)$ (cf. (1.4)), the product $\hat{Z}=\prod_{w \in W} w Z$ is clearly $W$-invariant. Since it follows from Chevalley's theorem that $S\left(\mathfrak{q}_{c}\right)^{H}$ is isomorphic to the subring of $S\left(\mathfrak{a}_{c}\right)$ consisting of $W$-invariant elements, we denote the element of $S\left(\mathfrak{q}_{c}\right)^{H}$ corresponding to $\hat{Z}$ by the same letter. On the other hand, let $e_{1}, \cdots, e_{p}$ be an arbitrary basis of $\mathfrak{a}^{\perp} \cap \mathfrak{q}$. Using the basis, we define $P=$ $\partial(\hat{Z})+\sum_{j=1}^{p} \tau\left(e_{j}\right)^{2}$. Let $u(X)$ be an ISH. Then, by definition, $u$ satisfies the differential equation $(P-c) u=0$ for some complex number $c$. Since $\sigma(P-c)=\sigma(P)$ does not vanish in a neighbourhood $U$ of $X_{0}$, we conclude that $u$ is real analytic on $U$. Hence the result follows.

q.e.d.

\section{§ 6. A condition on $q$-distinguished elements}

(6.1) Theorem 5.2 leads us to the following problem:

Problem A. Classify the symmetric pair $(\mathfrak{g}, \mathfrak{h})$ satisfying the condition $(\mathrm{C})$ :

(C) $\delta_{q}(Z)>0$ for any $\mathfrak{q}$-distinguished element $Z$ of $\mathfrak{q}$.

If the classification of $H$-orbits of $\mathscr{N}(\mathfrak{q})$ is accomplished, one would easily check the condition (C) for each $\mathfrak{q}$-distinguished nilpotent element. But the classification seems not to be done at present (cf. [S]). For this reason, we restrict our attention to look for examples of symmetric pairs which satisfy $(\mathrm{C})$.

(6.2) Before entering into treating the subject, we show that there exists a non-zero singular ISH for some symmetric pair. Hence consider the pair $(\mathfrak{g}, \mathfrak{h})=(\mathfrak{g} \mathfrak{D}(1, n+1), \mathfrak{g} \mathfrak{D}(1, n))$. In this case, $\mathfrak{q}$ is identified with the 
real vector space $V=\mathbf{R}^{n+1}$ with the canonical coordinate $x=\left(x_{0}, x_{1}, \cdots, x_{n}\right)$ and $H \simeq \mathrm{SO}_{0}(1, n)$. Then the action of $H$ on $\mathfrak{q}$ is identified with the natural action of $\mathrm{SO}_{0}(1, n)$ on $V$. In this case, the pseudo-Laplacian on $\mathfrak{q}$ coincides with

$$
P=-D_{x_{0}}^{2}+D_{x_{1}}^{2}+\cdots+D_{x_{n}}^{2}
$$

up to a constant factor. Then the system of differential equations introduced in (5.1) is rewritten in the following form for a certain complex number $\lambda$ :

$$
\mathscr{M}_{\lambda} \begin{cases}(P-\lambda) u=0 & \\ \left(x_{0} D_{x_{i}}+x_{i} D_{x_{0}}\right) u=0 & (i=1, \cdots, n) \\ \left(x_{i} D_{x_{j}}-x_{j} D_{x_{j}}\right) u=0 & (1 \leq i, j \leq n),\end{cases}
$$

Define $f(x)=-x_{0}^{2}+x_{1}^{2}+\cdots+x_{n}^{2}$. Then the nilpotent subvariety $\mathscr{N}(\mathfrak{q})$ of $\mathfrak{q}$ is identified with the set $\mathscr{N}(V)=\{x \in V ; f(x)=0\}$. It is easy to see that $V-\mathscr{N}(V)$ consists of $\mathfrak{q}$-regular semisimple elements and that $\mathscr{N}(V)-\{0\}$ consists of $\mathfrak{q}$-distinguished nilpotent elements. Take $X \in \mathscr{N}(V)-\{0\}$. Then it follows from direct calculation that $\mathfrak{q}_{X}=\mathbf{R} X$. This implies that $\delta_{\mathrm{a}}(X)=4-\operatorname{dim} V=3-n$. Hence if $n \geq 3$, the condition (C) does not hold. Moreover, if $n$ is odd and $n \geq 3$, there actually exists a singular ISH on $V$. In fact, consider the distribution $v_{s}(x)=|f(x)|_{+}^{s}$ on $V(s \in \mathbf{C})$. By definition, $v_{s}(x)$ coincides with $|f(x)|^{s}$ if $f(x) \geq 0$ and coincides with 0 if $f(x)<0$. Then it follows that $P v_{s}=4 s(s+(n-1) / 2) v_{s-1}$. Since $v_{s}$ is meromorphically dependent on $s$ and has a simple pole at $s=-(n-1) / 2$, one can put $u(x)=$ $\left.\left((s+(n-1) / 2) v_{s}(x)\right)\right|_{s=(1-n) / 2}$. Then it is clear that $u(x)$ satisfies the differential equations $\mathscr{M}_{\lambda}$ for $\lambda=0$. Moreover $\operatorname{Supp}(u)$ coincides with $\mathscr{N}(V)$. Hence $u(x)$ is a singular ISH. (Cf. [A])

In the sequel, we restrict our attention to look for examples of symmetric pairs satisfying the condition (C).

(6.3) In this paragraph, we give some known examples satisfying the condition $(\mathrm{C})$.

(6.3.1) Let $(\mathfrak{g}, \mathfrak{h})$ be a Riemannian symmetric pair. Then the assumption holds in this case. In fact, $\mathscr{N}(\mathfrak{q})=\{0\}$ holds in this case and moreover, any sub-symmetric pair of $(\mathfrak{g}, \mathfrak{h})$ is also Riemannian.

(6.3.2) Let $(\mathfrak{g}, \mathfrak{h})$ be a symmetric pair such that $\mathfrak{g}_{c}$ is identified with $\mathfrak{h}_{c} \oplus \mathfrak{h}_{c}$. In this case, $\left(\mathfrak{g}_{c}, \mathfrak{h}_{c}\right)$ is identified with $\left(\mathfrak{h}_{c} \oplus \mathfrak{h}_{c}, \mathfrak{h}_{c}\right)$. So one can check easily that the condition (C) holds for the pair (g, h) (cf. [A], [vD]).

(6.4) Let $(\mathfrak{g}, \mathfrak{h})$ be a symmetric pair and let $\left(\mathfrak{g}_{c}, \mathfrak{h}_{c}\right)$ be its complexi- 
fication. Let $X_{0}$ be an element of $\mathscr{N}\left(\mathfrak{q}_{c}\right)$, the nilpotent subvariety of $\mathfrak{q}_{c}$. Then one can define a number $\tilde{\delta}_{\mathrm{ac}}\left(X_{0}\right)$ quite similarly as the number introduced in Definition 1.11. The precise one is given as follows. Let $\left(A_{0}, X_{0}, Y_{0}\right)$ be a normal $S$-triple (cf. [KR]). Then ad $A_{0}$ leaves $\left(\mathfrak{q}_{c}\right)_{X_{0}}$ invariant. So we can choose a basis $w_{1}, \cdots, w_{r}$ of $\left(\mathfrak{q}_{c}\right)_{X_{0}}\left(r=\operatorname{dim}_{\mathrm{c}} \mathfrak{q}_{\mathrm{c}}\right)$ such that each $w_{i}$ is an eigenvector of ad $A_{0}$ (cf. (1.7)). Define the number $n_{i}$ by $\left[A_{0}, X_{0}\right]=n_{i} w_{i}$ for each $i$.

Definition 6.1. $\quad \tilde{\delta}_{\mathrm{q}_{c}}\left(X_{0}\right)=\sum_{i=1}^{r}\left(n_{i}+2\right)-\operatorname{dim}_{\mathrm{C}} \mathfrak{q}_{c}$.

It is clear from the definition that $\tilde{\delta}_{q_{c}}\left(X_{0}\right)=\delta_{q}\left(X_{0}\right)$ if $X_{0}$ is contained in $\mathscr{N}(\mathfrak{q})$. Note that $\mathscr{N}\left(\mathfrak{q}_{c}\right)$ always contains a $\mathfrak{q}_{c}$-regular element of $\mathfrak{q}_{c}$, that is, there exists a nilpotent element $X_{0} \in \mathfrak{q}_{c}$ such that $\operatorname{dim}_{\mathrm{c}}\left(\mathfrak{q}_{c}\right)_{X_{0}}=$ $\operatorname{dim}_{\mathrm{c}} \mathfrak{q}_{\mathrm{c}}-\operatorname{rank} \mathfrak{q}$. For any $Z \in \mathfrak{q}_{c}$, we also define the number $\tilde{\delta}_{\mathrm{q}_{c}}(Z)$ by the same way as we did in (1.10).

Lemma 6.2. Retain the notation above. Assume that $\mathrm{g}_{c}$ is simple. If $\tilde{\delta}_{q_{c}}\left(X_{0}\right)>0$ holds for any $\mathfrak{q}_{c}$-regular nilpotent element $X_{0}$ of $\mathfrak{q}_{c}$, then $\left(\mathfrak{g}_{c}, \mathfrak{h}_{c}\right)$ is isomorphic to one of the following pairs:

$$
\begin{aligned}
& \text { (I) }(\mathfrak{g l}(n, \mathbf{C}), \mathfrak{g} \mathfrak{D}(n, \mathbf{C})) \\
& \text { (II) } \quad(\mathfrak{g l}(2 n, \mathbf{C}), \mathfrak{g l}(n, \mathbf{C})+\mathfrak{g l}(n, \mathbf{C})+\mathbf{C}) \\
& \text { (III) } \quad(\mathfrak{g} \mathfrak{p}(n, \mathbf{C}), \mathfrak{H} l(n, \mathbf{C})+\mathbf{C}) \\
& \text { (IV) }\left(\mathfrak{g}_{\mathfrak{O}}(2 n+k, \mathbf{C}), \mathfrak{g}_{\mathfrak{O}}(n+k, \mathbf{C})+\mathfrak{g}_{\mathfrak{O}}(n, \mathbf{C})\right)(k=0,1,2) \\
& \text { (V) }\left(\mathrm{e}_{6}, \mathfrak{S p}_{\mathfrak{p}}(4, \mathrm{C})\right) \\
& \text { (VI) }\left(\mathfrak{e}_{6}, \mathfrak{g l}(6, \mathbf{C})+\mathfrak{I} \mathfrak{l}(2, \mathbf{C})\right) \\
& \text { (VII) } \quad\left(e_{7}, \mathfrak{s l}(8, \mathbf{C})\right) \\
& \text { (VIII) }\left(e_{8}, \mathfrak{g} \mathfrak{g}(16, \mathrm{C})\right) \\
& \text { (IX) } \quad\left(\mathfrak{f}_{4}, \mathfrak{S}_{\mathfrak{p}}(3, \mathbf{C})+\mathfrak{S l}_{(}(2, \mathbf{C})\right) \\
& \text { (X) } \quad\left(\mathfrak{g}_{2}, \mathfrak{g l}(2, \mathbf{C})+\mathfrak{g l}(2, \mathbf{C})\right) \\
& \text { (XI) }(\mathfrak{g} \mathfrak{D}(p+q, \mathbf{C}), \mathfrak{g} \mathfrak{D}(p, \mathbf{C})+\mathfrak{g} \mathfrak{D}(q, \mathbf{C}))(q+2<p<2 q+2) \text {. }
\end{aligned}
$$

This lemma is shown by using the classification of symmetric pairs and the results in (1.9) (cf. [He]).

Theorem 6.3. Let $\left(\mathfrak{g}_{c}, \mathfrak{h}_{c}\right)$ be a complex symmetric pair. Assume that $\left(\mathfrak{g}_{c}, \mathfrak{h}_{c}\right)$ is isomorphic to one of the pairs (I)-(X) in Lemma 6.2. Then $\tilde{\delta}_{a_{c}}(Z)$ $>0$ for any $\mathfrak{q}_{c}$-distinguished element $Z$ of $\mathfrak{q}_{c}$.

Remark. If $\left(\mathfrak{g}_{c}, \mathfrak{h}_{c}\right)$ is the pair in (XI), there exists a $\mathfrak{q}_{c}$-distinguished element $Z \in \mathfrak{q}_{c}$ such that $\tilde{\delta}_{q_{c}}(Z) \leq 0$.

To prove this theorem, we need a simple lemma.

Lemma 6.4. Let $\left(\mathfrak{g}_{c}, \mathfrak{h}_{c}\right)$ be a complex symmetric pair and let $\Sigma$ be its 
root system defined as in (1.4), that is, if $\left(\mathfrak{g}_{0}, \mathfrak{l}_{0}\right)$ is a Riemannian symmetric pair such that $\mathfrak{g}_{0}$ and $\mathfrak{f}_{0}$ are real forms of $\mathfrak{g}_{c}$ and $\mathfrak{h}_{c}$, respectively, then $\Sigma$ is the restricted root system of $g_{0}$. Assume that $\operatorname{dim}_{\mathrm{c}} \mathfrak{g}_{c}^{\alpha}+\operatorname{dim}_{\mathrm{c}} \mathfrak{g}_{c}^{2 \alpha} \leq 2$ for all $\alpha \in \Sigma$. Then each irreducible factor of $\left(\mathfrak{g}_{c}, \mathfrak{h}_{c}\right)$ is isomorphic to one of the pairs in $(\mathrm{I})-(\mathrm{X})$ or the pair $\left(\mathfrak{g}_{c}^{\prime} \oplus \mathfrak{g}_{c}^{\prime}, \mathfrak{g}_{c}^{\prime}\right)$ for some complex simple Lie algebra $g_{c}^{\prime}$.

Proof. It is clear that there exists a Riemannian symmetric pair $(\mathfrak{g}, \mathfrak{f})$ such that $\mathrm{g}$ is a real form of $\mathfrak{g}_{c}$ and $\mathfrak{f}$ is that of $\mathfrak{h}_{c}$. Then $\Sigma$ is nothing but the restricted root system of $g$. Then the lemma follows from Table in [W, p. 33].

Corollary 6.5. Let $\left(\mathfrak{g}_{c}, \mathfrak{h}_{c}\right)$ be a complex symmetric pair and let $\left(\mathfrak{g}_{c}^{\prime}, \mathfrak{h}_{c}^{\prime}\right)$ be its sub-symmetric pair. If $\left(\mathfrak{g}_{c}, \mathfrak{h}_{c}\right)$ satisfies the assumption of Lemma 6.4 , so does $\left(\mathfrak{g}_{c}^{\prime}, \mathfrak{h}_{c}^{\prime}\right)$.

By means of Corollary 6.5, to prove Theorem 6.3, it suffices to show the following.

Lemma 6.6. Retain the notation and the assumption in Theorem 6.3. Then $\tilde{\delta}_{\mathfrak{q}_{c}}(X)>0$ for all $\mathfrak{q}_{c}$-distinguished nilpotent element $X \in \mathfrak{q}_{c}$.

(6.5) We are going to prove Lemma 6.6 for the cases (I)-(X), separately.

(6.5.1) Proof for the case (I).

Let $\left(\mathfrak{g}_{c}, \mathfrak{h}_{c}\right)$ be a symmetric pair isomorphic to $(\mathfrak{I l}(n, \mathbf{C}), \mathfrak{g} \mathfrak{0}(n, \mathbf{C}))$ for some integer $n$. It is easy to see that, in this case, every $\mathfrak{q}_{c}$-distinguished nilpotent element is $g_{c}$-regular. Hence the claim for this case follows from Lemma 6.2.

(6.5.2) Proof for the case (II).

(a) Let $g_{c}=\operatorname{gr}(2 n, C)$ and let $\sigma$ be the involution of $g_{c}$ defined by

$$
\sigma X=\left[\begin{array}{rr}
I_{n} & 0 \\
0 & -I_{n}
\end{array}\right] X\left[\begin{array}{rr}
I_{n} & 0 \\
0 & -I_{n}
\end{array}\right]\left(X \in \mathfrak{g}_{c}\right) .
$$

Then

$$
\begin{aligned}
& \mathfrak{h}_{c}=\left\{\left[\begin{array}{ll}
A & 0 \\
0 & B
\end{array}\right] ; A, B \in \mathfrak{g} \mathfrak{l}(n, \mathbf{C}), \operatorname{tr}(A+B)=0\right\} \\
& \mathfrak{q}_{c}=\left\{\left[\begin{array}{ll}
0 & A \\
B & 0
\end{array}\right] ; A, B \in \mathfrak{g} \mathfrak{l}(n, \mathbf{C})\right\} .
\end{aligned}
$$

Moreover, define 


$$
H_{c}=\left\{\left[\begin{array}{ll}
A & 0 \\
0 & B
\end{array}\right] ; A, B \in \mathrm{GL}(n, \mathbf{C}), \operatorname{det}(A B)=1\right\} .
$$

(b) For each positive integer $d$, define the matrices $I_{d}, J_{d}, K_{d}, L_{d}$ in the following way:

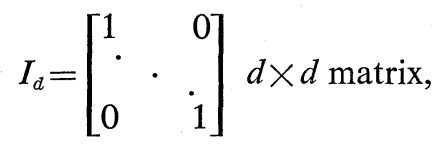

$$
\begin{aligned}
& J_{d}=\left[\begin{array}{llll}
0 & 1 & & \\
& 0 & \ddots & \\
& & \ddots & 1 \\
& & & 0
\end{array}\right] d \times d \text { matrix } \\
& K_{d}=\left[\begin{array}{lll}
1 & & 0 \\
& \ddots & \\
0 & 1 \\
0 & \cdots & 0
\end{array}\right](d+1) \times d \text { matrix, } \\
& L_{d}=\left[\begin{array}{ccc}
0 & 1 & \\
\vdots & \ddots & 0 \\
\vdots & 0 & \ddots \\
0 & & 1
\end{array}\right] d \times(d+1) \text { matrix. }
\end{aligned}
$$

(c) Let $\eta=\left(p_{1}, \cdots, p_{k}\right)$ be a partition of $2 n$, that is, each $p_{i}$ is an integer, $p_{1}+\cdots+p_{k}=2 n$ and $p_{1} \geq \cdots \geq p_{k}>0$.

Moreover, define subsets $I_{1}, I_{2}, I_{3}, I_{4}$ of $\{1, \cdots, k\}$ associated with the partition $\eta$ as follows:

(i) $\{1, \cdots, k\}=I_{1} \cup I_{2} \cup I_{3} \cup I_{4}$ (disjoint union)

(ii) $p_{i}$ is even for all $i \in I_{1} \cup I_{2}$.

(iii) $p_{i}$ is odd for all $i \in I_{3} \cup I_{4}$.

(iv) $\left|I_{3}\right|=\left|I_{4}\right|$.

Let $\mathscr{Q}_{n}$ be the totality of $\left(\eta,\left\{I_{1}, I_{2}, I_{3}, I_{4}\right\}\right)$ defined as above. Note that there are several possibilities of the choices of $\left\{I_{1}, I_{2}, I_{3}, I_{4}\right\}$ for a given partition $\eta$.

(d) For every $\zeta=\left(\eta,\left\{I_{1}, I_{2}, I_{3}, I_{4}\right\}\right) \in \mathscr{Q}_{n}\left(\eta=\left(p_{1}, \cdots, p_{k}\right)\right)$, define the matrix $X_{\zeta}$ as follows:

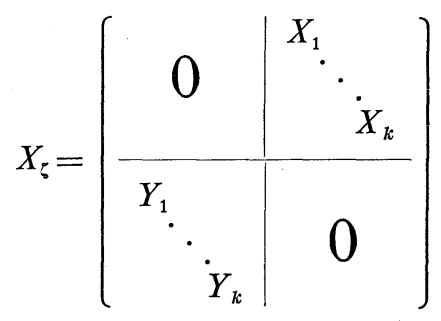


Here $X_{i}, Y_{j}$ are the matrices defined by

(Here $q_{i}=\left[p_{i} / 2\right]$.)

$$
X_{i}=\left\{\begin{array}{ll}
I_{q_{i}} & \left(i \in I_{1}\right) \\
J_{q_{i}} & \left(i \in I_{2}\right) \\
K_{q_{i}} & \left(i \in I_{3}\right) \\
L_{q_{i}} & \left(i \in I_{4}\right)
\end{array} \quad Y_{i}= \begin{cases}J_{q_{i}} & \left(i \in I_{1}\right) \\
I_{q_{i}} & \left(i \in I_{2}\right) \\
L_{q_{i}} & \left(i \in I_{3}\right) \\
K_{q_{i}} & \left(i \in I_{4}\right) .\end{cases}\right.
$$

(e) It is clear from the definition that for each $\zeta \in \mathscr{Q}_{n}, X_{\zeta}$ is nilpotent and is contained in $\mathfrak{q}_{c}$. Moreover, the following lemma is a direct consequence of $[\mathrm{S}, \S 3]$.

Lemma 6.7. For any nilpotent element $X$ of $\mathfrak{q}_{c}$, there exist $h \in H_{c}$ and $\zeta \in \mathscr{Q}_{n}$ such that $h X h^{-1}=X_{\zeta}$.

As to $\mathfrak{q}_{c}$-distinguished nilpotent elements of $\mathfrak{q}_{c}$, one can easily find the following lemma.

Lemma 6.8. Let $\zeta=\left(\eta,\left\{I_{1}, I_{2}, I_{3}, I_{4}\right\}\right) \in \mathscr{Q}_{n}$ and retain the notation in (d). Then $X_{\zeta}$ is $\mathfrak{q}_{c}$-distinguished nilpotent if and only if $X_{i}=X_{j}$ and $Y_{i}=$ $Y_{j}$ for every pair of indices $(i, j)$ such that $p_{i}=p_{j}$.

(f) Take $\zeta \in \mathscr{Q}_{n}$ and retain the notation in (d). Let $\left(A_{\zeta}, X_{\zeta}, Y_{\zeta}\right)$ be a normal $S$-triple. It is always possible to take $A_{\zeta}$ as a diagonal matrix and moreover such an $A_{\zeta}$ is uniquely determined. So we assume such an $A_{\zeta}$ is taken.

Let $X=\left[\begin{array}{ll}0 & Z \\ W & 0\end{array}\right] \in \mathfrak{q}_{c}$. We use the notation:

$Z=\left(Z_{i j}\right)_{1 \leq i, j \leq k}$, where $Z_{i j}$ is an $r_{i} \times s_{j}$ matrix

$W=\left(W_{i j}\right)_{1 \leq i, j \leq k}$, where $W_{i j}$ is an $s_{i} \times r_{j}$ matrix.

$\left(\right.$ Here $r_{i}=\left\{\begin{array}{ll}q_{i} & \text { if } i \in I_{1} \cup I_{2} \cup I_{4}, \\ q_{i}+1 & \text { if } i \in I_{3}\end{array} \quad s_{i}=\left\{\begin{array}{ll}q_{i} & \text { if } i \in I_{1} \cup I_{2} \cup I_{3} \\ q_{i}+1 & \text { if } i \in I_{4}\end{array}\right)\right.$.

Then define

$$
\begin{aligned}
& U_{i j}=\left\{X=\left[\begin{array}{rr}
0 & Z \\
W & 0
\end{array}\right] \in \mathfrak{q}_{c} ; Z_{\alpha \beta}=0, W_{\alpha \beta}=0 \text { if }(\alpha, \beta) \neq(i, j)\right\} \\
& V_{i j}=\left\{X \in U_{i j} ;\left[X, X_{\zeta}\right]=0\right\} .
\end{aligned}
$$

It follows from the definition that

$$
\left(\mathfrak{q}_{c}\right)_{X_{\zeta}}=\bigoplus_{i, j=1}^{k} V_{i j}
$$




$$
\operatorname{dim} U_{i j}=r_{i} s_{j}+r_{j} s_{i} .
$$

Now take a basis $w_{i j}^{1}, \cdots, w_{i j}^{n_{i j}}$ of $V_{i j}\left(n_{i j}=\operatorname{dim} V_{i j}\right)$ in such a way that $\left[A_{\zeta}, w_{i j}^{\alpha}\right]=f_{i j}^{\alpha} w_{i j}^{\alpha}\left(\alpha=i, \cdots, n_{i j}\right)$, where each $f_{i j}^{\alpha} \geq 0$ is an integer (cf. (1.7)). Using the notation introduced above, we define

$$
\chi_{i j}=\sum_{\alpha=1}^{n_{i j}}\left(f_{i j}^{\alpha}+2\right)-\operatorname{dim} V_{i j} .
$$

Then it is clear from the definition that

$$
\tilde{\delta}_{a_{c}}\left(X_{\zeta}\right)=\sum_{i, j=1}^{k} \chi_{i j}
$$

\section{Lemma 6.9.}

(i) $\quad \chi_{i i}= \begin{cases}p_{i} / 2 & \left(i \in I_{1} \cup I_{2}\right) \\ 0 & \left(i \in I_{3} \cup I_{4}\right) .\end{cases}$

(ii) If $i, j \in I_{1}(i \neq j)$ or if $i, j \in I_{2}(i \neq j)$, then $\chi_{i j}=\chi_{j i}=\min \left(p_{i}, p_{j}\right)$.

(iii) If $i \in I_{1}, j \in I_{2}$ or if $i \in I_{2}, j \in I_{1}$, then $\chi_{i j}=\chi_{j i}=0$.

(iv) If $i, j \in I_{3}(i<j)$ or if $i, j \in I_{4}(i<j)$, then $\chi_{i j}=\chi_{j i}=\left(p_{j}-p_{i}\right) / 2$.

(v) If $i \in I_{3}, j \in I_{4}$ or if $i \in I_{4}, j \in I_{3}$, then $\chi_{i j}=\chi_{j i}=\left(p_{i}+p_{j}+2\right) / 2$.

(vi) If $i \in I_{1} \cup I_{2}, j \in I_{3} \cup I_{4}$ or if $i \in I_{3} \cup I_{4}, j \in I_{1} \cup I_{2}$, then $\chi_{i j}+\chi_{j i}=$ $\min \left(p_{i}, p_{j}\right)$.

Since the proof of this lemma is elementary but rather lengthy, we omit it.

It follows from Lemma 6.9 that if $(i, j)$ satisfies one of the conditions in (i)-(iii), (v), (vi) of the lemma, then $\chi_{i j}+\chi_{j i} \geq 0$. On the other hand, if $(i, j)$ satisfies the condition in Lemma 6.9, (iv), then $\chi_{i j}+\chi_{j i} \leq 0$.

We now calculate the sum $S$ :

$$
\begin{aligned}
S= & \left(\sum_{i, j \in I_{3}, i<j}+\sum_{i, j \in I_{4}, i<j}\right)\left(\chi_{i j}+\chi_{j i}\right) \\
& +\sum_{i \in I_{3}, j \in I_{4}}\left(\chi_{i j}+\chi_{j i}\right) .
\end{aligned}
$$

Put $d=\left|I_{3}\right|=\left|I_{4}\right|$ and assume that $I_{3}=\left\{i_{1}, \cdots, i_{d}\right\}\left(i_{1}<\cdots<i_{d}\right)$ and $I_{4}=$ $\left\{j_{1}, \cdots, j_{d}\right\}\left(i_{1}<\cdots<j_{d}\right)$. Then it follows from Lemma 6.9 that

$$
\begin{aligned}
S & =\sum_{1 \leq \alpha<\beta \leq d}\left\{\left(\chi_{i_{\alpha} i_{\beta}}+\chi_{i_{\beta} i_{\alpha}}\right)+\left(\chi_{j_{\alpha} j_{\beta}}+\chi_{j_{\beta} j_{\alpha}}\right)\right\}+\sum_{\alpha=1}^{d} \sum_{\beta=1}^{d}\left(\chi_{i_{\alpha} j_{\beta}}+\chi_{j_{\beta} i_{\alpha}}\right) \\
& =\sum_{1 \leq \alpha<\beta \leq d}\left\{\left(p_{i_{\beta}}-p_{i_{\alpha}}\right)+\left(p_{j_{\beta}}-p_{j_{\alpha}}\right)\right\}+\sum_{\alpha=1}^{d} \sum_{\beta=1}^{d}\left(p_{i_{\alpha}}+p_{j_{\beta}}+2\right) \\
& =\sum_{\alpha=1}^{d}(2 \alpha-d-1)\left(p_{i_{\alpha}}+p_{j_{\beta}}\right)+d \sum_{\alpha=1}^{d}\left(p_{i_{\alpha}}+p_{j_{\beta}}\right)+2 d^{2}
\end{aligned}
$$




$$
=\sum_{\alpha=1}^{d}(2 \alpha-1)\left(p_{i_{\alpha}}+p_{j_{\alpha}}\right)+2 d^{2} .
$$

Hence we find that $S \geq 0$. If $d>0$, this implies that $\tilde{\delta}_{a_{0}}(X)>0$. On the other hand, if $d=0$, then $S=0$. In this case, $I_{1} \cup I_{2} \neq \emptyset$ and $\chi_{i i}>0$ for all $i \in I_{1} \cup I_{2}$ (cf. Lemma 6.9 (i)). This combined with the formula (6.5.2.1) implies that $\tilde{\delta}_{\mathrm{qc}}(X)>0$.

We have thus shown the next lemma which implies the conclusion of Lemma 6.6 for the case (II).

Lemma 6.10. $\quad \tilde{\delta}_{\mathrm{q}_{c}}(X)>0$ for all nilpotent element $X \in \mathfrak{q}_{c}$.

(6.5.3) Let $\left(\mathfrak{g}_{c}, \mathfrak{h}_{c}\right)$ be a symmetric pair isomorphic to one of the pairs in (III) and (IV). Then one can prove the statement similar to Lemma 6.10 by an argument similar to that in (6.5.2). Hence Lemma 6.6 is true for the cases (III) and (IV).

\section{(6.5.4) Proof for the cases (V)-(X).}

To prove the conclusion of Lemma 6.6 for the cases (V)-(X), we use the classification of the nilpotent orbits of exceptional simple Lie algebras by Dynkin [D].

From now on, $\mathfrak{g}$ denotes a complex simple Lie algebra instead of real one for the sake of simplicity. Let $X$ be a nilpotent element of $\mathrm{g}$ and let $(A, X, Y)$ be an $S$-triple. Then we can choose a basis $w_{1}, \cdots, w_{p}$ of $\mathfrak{g}_{X}$ ( $p=\operatorname{dim} \mathrm{g}_{X}$ ) such that each of $w_{1}, \cdots, w_{p}$ is an eigenvector of $\operatorname{ad}(A)$. So put $\left[A, w_{i}\right]=n_{i} w_{i}(1 \leq i \leq p)$. Then $n_{1}, \cdots, n_{p}$ are non-negative integers. We may assume without loss of generality that $n_{1} \geq \cdots \geq n_{k}>n_{k+1}=\cdots$ $=n_{p}=0$. Let $\sigma$ be a complex linear involution of $\mathfrak{g}$ and let $\mathfrak{h}$ and $\mathfrak{q}$ be the 1-eigenspace and the $(-1)$-eigenspace of $\sigma$, respectively. Now assume that $X$ is contained in $\mathfrak{q}$ and $(A, X, Y)$ is a normal $S$-triple. Then we may assume from the first that each of $w_{1}, \cdots, w_{p} \in \mathfrak{g}_{w}$ is contained in $\mathfrak{h}$ or $\mathfrak{q}$. At this moment, there are two possibilities:

(a) $\operatorname{dim} \mathfrak{h}_{X}>p-k$.

(b) $\operatorname{dim} \mathfrak{h}_{X} \leq p-k$.

In the case (a), $X$ is clearly not $\mathfrak{q}$-distinguished (cf. Lemma 1.12). On the other hand, in the case (b), we define

$$
n(X)=\sum_{i=k-q+1}^{k}\left(n_{i}+2\right)-\operatorname{dim} \mathfrak{q} \quad\left(q=\operatorname{dim} \mathfrak{q}_{X}\right) .
$$

If $X$ is $\mathfrak{q}$-distinguished, it is clear from the definition that $\tilde{\delta}_{q}(X) \geq n(X)$. Then we obtain the next lemma from Tables I-V in an Appendix.

Lemma 6.11. Let $(\mathfrak{g}, \mathfrak{h})$ be a complex symmetric pair. 
(i) If $(\mathfrak{g}, \mathfrak{h})$ is isomorphic to one of the pairs (V), (VII)-(X), then $n(X)>0$ for all $\mathfrak{q}$-distinguished nilpotent element $X$ of $\mathfrak{q}$.

(ii) Assume that $(\mathfrak{g}, \mathfrak{h})$ is the pair $(\mathrm{VI})$, namely, $(\mathfrak{g}, \mathfrak{h}) \simeq\left(\mathfrak{e}_{6}, \mathfrak{g l}(6, \mathbf{C})+\right.$ $\mathfrak{I}(2, \mathbf{C}))$. If $X$ is $\mathfrak{q}$-distinguished nilpotent and $n(X) \leq 0$, then the weighted Dynkin diagram $\triangle(X)$ of $X$ is one of the following ones:

$$
\begin{array}{cc}
22022, & 20202 \\
2 & 0
\end{array}
$$

We can also prove the next lemma. Since we need elementary but complicated arguments for the proof, we leave its proof in another paper.

Lemma 6.12. Retain the notation in Lemma 6.11 (ii). Let $X$ be a nilpotent element of $\mathfrak{q}$. Let $u_{1}, \cdots, u_{q}$ be a basis of $\mathfrak{q}_{x}$. Assume that $\left[A, u_{i}\right]=m_{i} u_{i}$, where $m_{i}$ is a non-negative integer $\left(m_{1} \geq \cdots \geq m_{q}\right)$.

(i) Assume that $\Delta(X)=22022$. Then $q=5$ and $\left(m_{1}, \cdots, m_{5}\right)=$ $(14,10,10,6,2)$.

(ii) Assume that $\Delta(X)=20202$. Then $q=7$ and $\left(m_{1}, \cdots, m_{7}\right)$ coin0 cides with $(10,10,6,6,2,2,2)$ or $(10,8,6,4,4,2,2)$.

The conclusion of Lemma 6.6 for the cases (V)-(X) follows from Lemma 6.11 and 6.12 .

Corollary to Theorem 6.3. Let $(\mathfrak{g}, \mathfrak{h})$ be a symmetric pair such that $\left(\mathfrak{g}_{c}, \mathfrak{h}_{c}\right)$ is one of the pairs in (I)-(X). Then $(\mathfrak{g}, \mathfrak{h})$ satisfies the assumption of Theorem 5.2.

\section{§ 7. Two conjectures}

In this section, we present two conjectures on the system $\mathscr{M}_{A}$ defined in (5.1.1). It is provable that $\mathscr{M}_{\Lambda}$ is holonomic for any $\Lambda \in \mathfrak{a}_{c}^{*}$.

Conjecture 7.1. $\mathscr{M}_{A}$ is regular holonomic in the sense of KashiwaraKawai (cf. [HK]).

If this is true, one would conclude that any ISH on $q$ turns out to be a distribution. This follows from the general theory of regular holonomic systems.

Conjecture 7.1 is true in some cases. For example, if $q$ is of rank one, one can easily show that $\mathscr{M}_{A}$ is regular holonomic. On the other hand, if the pair $(\mathfrak{g}, \mathfrak{h})$ is the one in (6.3.2), then $\mathscr{M}_{A}$ is also regular holonomic. This is a deep result shown in [HK]. To prove this, Hotta and Kashiwara [HK] showed that $\mathscr{M}_{A}$ coincides with the minimal extension of 
the restriction $\mathscr{M}_{A} \mid\left(\mathfrak{g}_{c}\right)^{\prime}$ to $\left(\mathfrak{g}_{c}\right)^{\prime}$ which is the totality of regular semisimple elements of $g_{c}$. This leads us to the next conjecture.

Conjecture 7.2. Let $(\mathfrak{g}, \mathfrak{h})$ be a symmetric pair. Assume that $\left(\mathfrak{g}_{c}, \mathfrak{h}_{c}\right)$ satisfies the assumption of Lemma 6.4. Then the system $\mathscr{M}_{A}$ coincides with the minimal extension of the restriction $\mathscr{M}_{A} \mid\left(\mathfrak{q}_{c}\right)^{\prime}$ to $\left(\mathfrak{q}_{c}\right)^{\prime}$ which is the totality of the $\mathfrak{q}_{c}$-regular semisimple elements of $\mathfrak{q}_{c}$.

One easily finds that the assumption of Conjecture 7.2 is necessary. In fact, consider the symmetric pair $\left(\mathfrak{g} 0(n+1,1), \mathfrak{g}_{\mathfrak{O}}(n, 1)\right)$. In the case where $n$ is odd and is greater than 2 , the system $\mathscr{M}_{1}$ with $\Lambda=0$ has a nontrivial coherent quotient whose support is contained in the outside of the set of regular semisimple elements of $\mathfrak{q}_{c}$. This follows from the arguments in (6.2).

\section{Appendix}

We give a proof of Lemma 6.11 in this appendix.

Retain the notation in Lemma"6.11. Let $X$ be a nilpotent element of $\mathrm{g}$ and let $\Delta(X)$ be its weighted Dynkin diagram. We use the notation: $p=\operatorname{dim}_{\mathrm{c}} \mathfrak{g}_{X}$ and $q=\operatorname{dim}_{\mathrm{C}} \mathfrak{q}_{X}$. Note that $p$ is determined in [E]. Also the number $q$ is easily determined from $p$ (cf. Corollary 1.15). Moreover one can decide the numbers $n_{1}, \cdots, n_{p}$ by using the weighted Dynkin diagram $\Delta(X)$ and the root system of $g$.

Noting these facts, we give the numbers $n(X)$ for all niloptent elements $X$ of $\mathfrak{g}$ in Tables I-V. Explain the contents of the Tables. In each table, the weighted Dynkin diagram, the numbers $n_{1}, \cdots, n_{p}, p=\operatorname{dim} \mathrm{g}_{X}, q=$ $\operatorname{dim} \mathfrak{q}_{X}, n=n(X)$ are arranged in order. In the case where $(a): \operatorname{dim} \mathfrak{h}_{X}>$ $p-k$ (cf. (6.3.4)) holds for the nilpotent element $X$, we write $N$ instead of the number $q$. In each Table, the notation $n^{d}$ means that the number $n$ occurs with multiplicity $d$.

Only in the case of Table I, we must remark one comment more. In this case, we treat the case of the simple Lie algebra of type $E_{6}$. This coorresponds to the pairs in (V) and (VI). The numbers $q$ and $n$ are used in the former case. On the other hand, $q^{\prime}$ and $n^{\prime}$ are used instead of $q$ and $n$ in the latter case.

Table I: $\left(\mathfrak{e}_{6}, \mathfrak{a p}(4, \mathrm{C})\right)$ and $\left(\mathfrak{e}_{6}, \mathfrak{g l}(6, \mathrm{C})+\mathfrak{a l}(2, \mathrm{C})\right)$

\begin{tabular}{ccccccc}
\hline$\Delta(X)$ & $n_{1}, \cdots, n_{p}$ & $p$ & $q$ & $n$ & $q^{\prime}$ & $n^{\prime}$ \\
\hline $\begin{array}{c}22222 \\
2\end{array}$ & $22,16,14,10,8,2$ & 6 & 6 & 42 & 4 & 2 \\
\hline $\begin{array}{c}22022 \\
2\end{array}$ & $16,14,10,10,8,6,4,2$ & 8 & 7 & 26 & 5 & 0 \\
\hline
\end{tabular}




\begin{tabular}{|c|c|c|c|c|c|c|}
\hline $\begin{array}{c}20202 \\
2 \\
\end{array}$ & $14,10^{3}, 8,6,4,4,2,0$ & 10 & 8 & 28 & 6 & 6 \\
\hline $\begin{array}{c}20202 \\
0\end{array}$ & $10,10,8,8,6,6,4^{3}, 2^{3}$ & 12 & 9 & 14 & 7 & -2 \\
\hline $\begin{array}{c}21012 \\
1 \\
\end{array}$ & $10,9^{2}, 8,6,5^{2}, 4,3^{2}, 2,0^{3}$ & 14 & 10 & 32 & 8 & 12 \\
\hline $\begin{array}{c}11011 \\
2\end{array}$ & $10,8,7^{2}, 6^{2}, 5^{2}, 4,2^{2}, 1^{2}, 0$ & 14 & 10 & 17 & 8 & 2 \\
\hline $\begin{array}{c}11011 \\
1\end{array}$ & $8,7^{2}, 6,5^{2}, 4^{3}, 3^{2}, 2^{2}, 1^{2}, 0$ & 16 & 11 & 14 & 9 & 2 \\
\hline $\begin{array}{c}00200 \\
2\end{array}$ & $10,6^{8}, 2,0^{8}$ & 18 & 12 & $\mathrm{~N}$ & 10 & 40 \\
\hline $\begin{array}{c}20002 \\
2\end{array}$ & $8,6^{5}, 4^{3}, 2^{5}, 0^{4}$ & 18 & 12 & 28 & 10 & 14 \\
\hline $\begin{array}{c}00200 \\
0\end{array}$ & $6^{2}, 4^{7}, 2^{9}, 0^{2}$ & 20 & 13 & 18 & 11 & 8 \\
\hline $\begin{array}{c}01010 \\
1\end{array}$ & $6,5^{2}, 4^{3}, 3^{6}, 2^{4}, 1^{2}, 0^{4}$ & 22 & 14 & 22 & 12 & 12 \\
\hline $\begin{array}{c}10101 \\
0\end{array}$ & $5^{2}, 4^{4}, 3^{4}, 2^{5}, 1^{6}, 0^{3}$ & 24 & 15 & 16 & 13 & 8 \\
\hline $\begin{array}{c}10001 \\
2\end{array}$ & $6,4^{5}, 3^{8}, 2,0^{11}$ & 26 & 16 & $\mathrm{~N}$ & 14 & 34 \\
\hline $\begin{array}{c}01010 \\
0\end{array}$ & $4^{3}, 3^{4}, 2^{9}, 1^{8}, 0^{4}$ & 28 & 17 & 18 & 15 & 12 \\
\hline $\begin{array}{c}20002 \\
0\end{array}$ & $4^{8}, 2^{8}, 0^{14}$ & 30 & 18 & $\mathrm{~N}$ & 16 & 40 \\
\hline $\begin{array}{c}10001 \\
1\end{array}$ & $4,3^{6}, 2^{8}, 1^{8}, 0^{9}$ & 32 & 19 & 24 & 17 & 21 \\
\hline $\begin{array}{c}00000 \\
2\end{array}$ & $4,2^{19}, 0^{16}$ & 36 & 21 & $\mathrm{~N}$ & 19 & 36 \\
\hline $\begin{array}{c}00100 \\
0\end{array}$ & $3^{2}, 2^{9}, 1^{16}, 0^{11}$ & 38 & 22 & 30 & 20 & 24 \\
\hline $\begin{array}{c}10001 \\
0\end{array}$ & $2^{8}, 1^{16}, 0^{22}$ & 46 & 26 & $\mathrm{~N}$ & 24 & 40 \\
\hline $\begin{array}{c}00000 \\
1 \\
\end{array}$ & $2,1^{20}, 0^{35}$ & 56 & 31 & $\mathrm{~N}$ & 29 & $\mathrm{~N}$ \\
\hline $\begin{array}{c}00000 \\
0\end{array}$ & $0^{78}$ & 78 & 42 & $\mathrm{~N}$ & 40 & $\mathrm{~N}$ \\
\hline
\end{tabular}


Table II: $\left(\mathfrak{e}_{7}, \widehat{B g}(8, \mathbf{C})\right)$

\begin{tabular}{|c|c|c|c|c|}
\hline$\Delta(X)$ & $n_{1}, \cdots, n_{p}$ & $p$ & $q$ & $n$ \\
\hline $\begin{array}{c}222222 \\
2\end{array}$ & $34,26,22,18,14,10,2$ & 7 & 7 & 70 \\
\hline $\begin{array}{c}220222 \\
2\end{array}$ & $26,22,18,16,14,10^{2}, 6,2$ & 9 & 8 & 44 \\
\hline $\begin{array}{c}220202 \\
2\end{array}$ & $22,18,16,14^{2}, 10^{2}, 8,6,2^{2}$ & 11 & 9 & 30 \\
\hline $\begin{array}{l}222020 \\
0\end{array}$ & $22,16^{3}, 14,10,8^{3}, 2,0^{3}$ & 13 & 10 & 70 \\
\hline $\begin{array}{c}202022 \\
0\end{array}$ & $18,16,14^{2}, 10^{3}, 8,6^{2}, 4,2^{2}$ & 13 & 10 & 22 \\
\hline $\begin{array}{c}210122 \\
1\end{array}$ & $18,15^{2}, 14,10^{2}, 9^{2}, 6,5^{2}, 2,0^{3}$ & 15 & 11 & 52 \\
\hline $\begin{array}{c}202020 \\
0\end{array}$ & $16,14,12^{2}, 10^{2}, 8^{3}, 6,4^{3}, 2,0$ & 15 & 11 & 28 \\
\hline $\begin{array}{l}202002 \\
0\end{array}$ & $14,12,10^{4}, 8^{2}, 6^{3}, 4^{2}, 2^{4}$ & 17 & 12 & 14 \\
\hline $\begin{array}{c}210102 \\
1\end{array}$ & $14,11^{2}, 10^{2}, 9^{2}, 8,6^{2}, 5^{2}, 3^{2}, 2^{2}, 0^{3}$ & 19 & 13 & 34 \\
\hline $\begin{array}{c}210110 \\
1\end{array}$ & $14,11^{2}, 10,9^{2}, 8^{3}, 6,5^{2}, 3^{2}, 2^{2}, 0^{3}$ & 19 & 13 & 34 \\
\hline $\begin{array}{c}002020 \\
0\end{array}$ & $12^{3}, 10,8^{3}, 6^{5}, 4^{3}, 2,0^{3}$ & 19 & 13 & 34 \\
\hline $\begin{array}{c}220020 \\
0\end{array}$ & $14,10^{5}, 8^{3}, 6,4^{4}, 2,0^{6}$ & 21 & 14 & 56 \\
\hline $\begin{array}{c}002002 \\
0\end{array}$ & $10^{3}, 8^{3}, 6^{5}, 4^{4}, 2^{6}$ & 21 & 14 & 10 \\
\hline $\begin{array}{c}010102 \\
1\end{array}$ & $10^{2}, 9,8^{2}, 7^{2}, 6^{3}, 5^{3}, 3^{4}, 2^{3}, 0^{3}$ & 23 & 15 & 25 \\
\hline $\begin{array}{c}020020 \\
0\end{array}$ & $10^{2}, 8^{4}, 6^{4}, 4^{6}, 2^{5}, 0^{2}$ & 23 & 15 & 18 \\
\hline $\begin{array}{c}101012 \\
0\end{array}$ & $10,9^{2}, 8^{3}, 6^{2}, 5^{4}, 4^{2}, 3^{3}, 2^{2}, 1^{3}, 0^{3}$ & 25 & 16 & 18 \\
\hline $\begin{array}{c}101020 \\
0\end{array}$ & $10,9^{2}, 8^{3}, 6,5^{6}, 4^{3}, 3^{2}, 2,0^{6}$ & 25 & 16 & 42 \\
\hline $\begin{array}{c}200200 \\
0\end{array}$ & $10,8^{3}, 6^{8}, 4^{3}, 2^{7}, 0^{5}$ & 25 & 16 & 24 \\
\hline $\begin{array}{c}201010 \\
0\end{array}$ & $10,8,7^{4}, 6^{4}, 5^{4}, 4,2^{4}, 1^{4}, 0^{4}$ & 27 & 17 & 24 \\
\hline
\end{tabular}




\begin{tabular}{|c|c|c|c|c|}
\hline $\begin{array}{c}002000 \\
0\end{array}$ & $8^{3}, 6^{5}, 4^{10}, 2^{6}, 0^{3}$ & 27 & 17 & 22 \\
\hline $\begin{array}{c}101010 \\
0\end{array}$ & $8,7^{2}, 6^{3}, 5^{4}, 4^{5}, 3^{4}, 2^{4}, 1^{4}, 0^{2}$ & 29 & 18 & 15 \\
\hline $\begin{array}{c}200022 \\
0\end{array}$ & $10,8^{7}, 6,4^{7}, 2,0^{14}$ & 31 & 19 & $\mathrm{~N}$ \\
\hline $\begin{array}{c}210001 \\
1\end{array}$ & $10,7^{4}, 6^{6}, 5^{4}, 2^{2}, 1^{4}, 0^{10}$ & 31 & 19 & 53 \\
\hline $\begin{array}{c}200020 \\
0\end{array}$ & $8,6^{7}, 4^{9}, 2^{7}, 0^{9}$ & 33 & 20 & 44 \\
\hline $\begin{array}{c}000200 \\
0\end{array}$ & $6^{5}, 4^{10}, 2^{15}, 0^{3}$ & 33 & 20 & 20 \\
\hline $\begin{array}{c}001010 \\
0\end{array}$ & $6^{3}, 5^{4}, 4^{4}, 3^{8}, 2^{8}, 1^{4}, 0^{4}$ & 35 & 21 & 20 \\
\hline $\begin{array}{c}010001 \\
1\end{array}$ & $6^{2}, 5^{4}, 4^{5}, 3^{8}, 2^{8}, 1^{4}, 0^{6}$ & 37 & 22 & 26 \\
\hline $\begin{array}{c}220000 \\
0\end{array}$ & $10,6^{14}, 2,0^{21}$ & 37 & 22 & $\mathrm{~N}$ \\
\hline $\begin{array}{c}100101 \\
0\end{array}$ & $6,5^{4}, 4^{7}, 3^{6}, 2^{7}, 1^{8}, 0^{5}$ & 39 & 23 & 24 \\
\hline $\begin{array}{c}020000 \\
0\end{array}$ & $6^{2}, 4^{13}, 2^{15}, 0^{9}$ & 39 & 23 & 38 \\
\hline $\begin{array}{c}101000 \\
0\end{array}$ & $6,5^{2}, 4^{7}, 3^{10}, 2^{6}, 1^{6}, 0^{9}$ & 41 & 24 & 34 \\
\hline $\begin{array}{c}010010 \\
0\end{array}$ & $5^{2}, 4^{6}, 3^{8}, 2^{11}, 1^{10}, 0^{6}$ & 43 & 25 & 24 \\
\hline $\begin{array}{c}200002 \\
0\end{array}$ & $6,4^{15}, 2^{10}, 0^{21}$ & 47 & 27 & $\mathrm{~N}$ \\
\hline $\begin{array}{l}200010 \\
0\end{array}$ & $6,4^{7}, 3^{16}, 2,0^{24}$ & 49 & 28 & $\mathrm{~N}$ \\
\hline $\begin{array}{c}000020 \\
0\end{array}$ & $4^{10}, 2^{22}, 0^{17}$ & 49 & 28 & 54 \\
\hline $\begin{array}{c}000000 \\
2\end{array}$ & $47,2^{28}, 0^{14}$ & 49 & 28 & 42 \\
\hline $\begin{array}{c}001000 \\
0\end{array}$ & $4^{3}, 3^{8}, 2^{15}, 1^{16}, 0^{9}$ & 51 & 29 & 30 \\
\hline $\begin{array}{c}100010 \\
0\end{array}$ & $4,3^{8}, 2^{16}, 1^{16}, 0^{16}$ & 57 & 32 & 42 \\
\hline $\begin{array}{c}000001 \\
1\end{array}$ & $3^{6}, 2^{16}, 1^{20}, 0^{21}$ & 63 & 35 & 50 \\
\hline
\end{tabular}




\begin{tabular}{clllll}
\hline $\begin{array}{c}200000 \\
0\end{array}$ & $4,2^{31}, 0^{24}$ & 67 & 37 & $\mathrm{~N}$ \\
\hline $\begin{array}{c}010000 \\
0\end{array}$ & $3^{2}, 2^{15}, 1^{28}, 0^{24}$ & 69 & 38 & 54 \\
\hline $\begin{array}{c}000002 \\
0\end{array}$ & $2^{27}, 0^{52}$ & 79 & 43 & $\mathrm{~N}$ \\
\hline $\begin{array}{c}000010 \\
0\end{array}$ & $2^{10}, 1^{32}, 0^{39}$ & 81 & 44 & $\mathrm{~N}$ \\
\hline $\begin{array}{c}100000 \\
0\end{array}$ & $2,1^{32}, 0^{66}$ & 99 & 53 & $\mathrm{~N}$ \\
\hline $\begin{array}{c}000000 \\
0\end{array}$ & $0^{133}$ & 133 & 70 & $\mathrm{~N}$ \\
\hline
\end{tabular}

Table III: $\quad\left(e_{8}, \mathfrak{g}_{\mathfrak{b}}(16, \mathrm{C})\right)$

\begin{tabular}{|c|c|c|c|c|}
\hline$\Delta(X)$ & $n_{1}, \cdots, n_{p}$ & $p$ & $q$ & $n$ \\
\hline $\begin{array}{l}2222222 \\
2\end{array}$ & $58,46,38,34,26,22,14,2$ & 8 & 8 & 128 \\
\hline $\begin{array}{l}2202222 \\
2\end{array}$ & $46,38,34,28,26,22,18,14,10,2$ & 10 & 9 & 82 \\
\hline $\begin{array}{c}2202022 \\
2 \\
\end{array}$ & $38,34,28,26,22^{2}, 18,16,14,10,6,2$ & 12 & 10 & 56 \\
\hline $\begin{array}{l}2020222 \\
0\end{array}$ & $34,28,26^{2}, 22,18^{2}, 16,14,10^{2}, 8,2^{2}$ & 14 & 11 & 40 \\
\hline $\begin{array}{c}2101222 \\
1\end{array}$ & $34,27^{2}, 26,22,18,17^{2}, 14,10,9^{2}, 2,0^{3}$ & 16 & 12 & 100 \\
\hline $\begin{array}{c}2020202 \\
0\end{array}$ & $28,26,22^{2}, 18^{2}, 16,14^{3}, 10^{2}, 8,6,4,2$ & 16 & 12 & 30 \\
\hline $\begin{array}{c}2020022 \\
0\end{array}$ & $26,22^{2}, 20,18,16^{2}, 14^{2}, 12,10^{3}, 6^{2}, 4,2^{2}$ & 18 & 13 & 20 \\
\hline $\begin{array}{c}2101022 \\
1\end{array}$ & $26,22,21^{2}, 18,16,15^{2}, 14,11^{2}, 10^{2}, 6,5^{2}, 2,0^{3}$ & 20 & 14 & 59 \\
\hline $\begin{array}{l}2020020 \\
0\end{array}$ & $22^{2}, 20,18,16,14^{3}, 12^{2}, 10^{4}, 8,6,4,2^{3}$ & 20 & 14 & 16 \\
\hline $\begin{array}{l}2101101 \\
1\end{array}$ & $22,21^{2}, 18,15^{2}, 14,12^{3}, 11^{2}, 10,9^{2}, 6,3^{2}, 2,0^{3}$ & 22 & 15 & 46 \\
\hline $\begin{array}{c}0020022 \\
0\end{array}$ & $22,18^{2}, 16^{3}, 14^{3}, 10^{3}, 8^{3}, 6^{2}, 4,2^{4}$ & 22 & 15 & 8 \\
\hline $\begin{array}{l}0101022 \\
1 \\
\end{array}$ & $22,18,17^{2}, 16,15^{2}, 14^{2}, 10^{2}, 9^{2}, 8,7^{2}, 6,3^{2}, 2^{2}, 0^{3}$ & 24 & 16 & 38 \\
\hline
\end{tabular}




\begin{tabular}{|c|c|c|c|c|}
\hline $\begin{array}{l}0020020 \\
0\end{array}$ & $18^{2}, 16,14^{3}, 12^{3}, 10^{3}, 8^{3}, 6^{5}, 4,2^{3}$ & 24 & 16 & 10 \\
\hline $\begin{array}{l}1010122 \\
0\end{array}$ & $22,17^{2}, 16^{3}, 15^{2}, 14,10,9^{2}, 8^{3}, 7^{2}, 2^{2}, 1^{4}, 0^{3}$ & 26 & 17 & 24 \\
\hline $\begin{array}{l}2002002 \\
0\end{array}$ & $18,16^{2}, 14^{3}, 12,10^{6}, 8^{3}, 6^{3}, 4^{2}, 2^{4}, 0$ & 26 & 17 & 14 \\
\hline $\begin{array}{l}2010102 \\
0\end{array}$ & $18,16,15^{2}, 14^{2}, 11^{2}, 10^{3}, 9^{4}, 8,6^{2}, 5^{2}, 4,2^{2}, 1^{2}, 0^{3}$ & 28 & 18 & 25 \\
\hline $\begin{array}{l}0020002 \\
0\end{array}$ & $16,14^{3}, 12^{2}, 10^{6}, 8^{3}, 6^{5}, 4^{4}, 2^{4}$ & 28 & 18 & 6 \\
\hline $\begin{array}{l}1010102 \\
0\end{array}$ & $16,14,13^{2}, 12^{2}, 11^{2}, 10^{2}, 9^{2}, 8^{3}, 7^{2}, 6,5^{2}, 4^{3}, 3^{2}, 2^{2}, 1^{2}, 0$ & 30 & 19 & 6 \\
\hline $\begin{array}{l}1010110 \\
0\end{array}$ & $15^{2}, 14,12^{3}, 11^{2}, 10,9^{2}, 8^{3}, 7^{4}, 6,5^{2}, 4^{3}, 3^{2}, 2,0^{3}$ & 30 & 19 & 26 \\
\hline $\begin{array}{l}2000222 \\
0\end{array}$ & $22,16^{7}, 14,10,8^{7}, 2,0^{14}$ & 32 & 20 & $\mathbf{N}$ \\
\hline $\begin{array}{c}2100012 \\
1\end{array}$ & $18,15^{4}, 14,10^{6}, 9^{4}, 6,5^{4}, 2,0^{10}$ & 32 & 20 & 95 \\
\hline $\begin{array}{l}1010101 \\
0\end{array}$ & $14,13^{2}, 12,11^{2}, 10^{2}, 9^{2}, 8^{3}, 7^{4}, 6^{3}, 5^{2}, 4^{3}, 3^{2}, 2^{2}, 1^{2}, 0$ & 32 & 20 & 8 \\
\hline $\begin{array}{l}2000202 \\
0\end{array}$ & $16,14,12^{6}, 10^{2}, 8^{7}, 6,4^{7}, 2,0^{8}$ & 34 & 21 & 62 \\
\hline $\begin{array}{l}0002002 \\
0\end{array}$ & $14,12^{2}, 10^{7}, 8^{5}, 6^{5}, 4^{5}, 2^{8}, 0$ & 34 & 21 & 4 \\
\hline $\begin{array}{l}0010102 \\
0\end{array}$ & $14,12,11^{2}, 10^{4}, 9^{4}, 8^{2}, 7^{2}, 6^{3}, 5^{2}, 4^{2}, 3^{4}, 2^{4}, 1^{2}, 0^{3}$ & 36 & 22 & 13 \\
\hline $\begin{array}{l}1010100 \\
0\end{array}$ & $12^{3}, 11^{2}, 10,9^{2}, 8^{3}, 7^{4}, 6^{5}, 5^{4}, 4^{3}, 3^{2}, 2^{2}, 1^{2}, 0^{3}$ & 36 & 22 & 18 \\
\hline $\begin{array}{l}0100012 \\
1\end{array}$ & $14,11^{4}, 10^{2}, 9^{4}, 8^{5}, 6^{2}, 5^{4}, 3^{4}, 2^{6}, 0^{6}$ & 38 & 23 & 32 \\
\hline $\begin{array}{c}2000200 \\
0\end{array}$ & $12^{3}, 10^{5}, 8^{3}, 6^{13}, 4^{3}, 2^{5}, 0^{6}$ & 38 & 23 & 34 \\
\hline $\begin{array}{l}1001012 \\
0\end{array}$ & $14,11^{2}, 10^{5}, 9^{4}, 8^{3}, 7^{2}, 6,5^{2}, 4^{4}, 3^{2}, 2^{2}, 1^{6}, 0^{6}$ & 40 & 24 & 24 \\
\hline $\begin{array}{l}0002000 \\
0\end{array}$ & $10^{4}, 8^{6}, 6^{10}, 4^{10}, 2^{10}$ & 40 & 24 & 4 \\
\hline $\begin{array}{l}0010100 \\
0\end{array}$ & $10^{3}, 9^{2}, 8^{3}, 7^{4}, 6^{5}, 5^{6}, 4^{4}, 3^{6}, 2^{6}, 0^{3}$ & 42 & 25 & 16 \\
\hline $\begin{array}{c}0100010 \\
1\end{array}$ & $10^{2}, 9^{4}, 8,7^{4}, 6^{7}, 5^{4}, 4^{5}, 3^{8}, 2^{3}, 0^{6}$ & 44 & 26 & 30 \\
\hline
\end{tabular}




\begin{tabular}{|c|c|c|c|c|}
\hline $\begin{array}{l}1001010 \\
0\end{array}$ & $10^{2}, 9^{2}, 8^{4}, 7^{4}, 6^{4}, 5^{6}, 4^{7}, 3^{4}, 2^{4}, 1^{4}, 0^{3}$ & 44 & 26 & 12 \\
\hline $\begin{array}{l}1010001 \\
0\end{array}$ & $10,9^{4}, 8^{3}, 7^{2}, 6^{5}, 5^{8}, 4^{7}, 3^{4}, 2^{2}, 1^{4}, 0^{6}$ & 46 & 27 & 26 \\
\hline $\begin{array}{l}0100101 \\
0 \\
\end{array}$ & $10,9^{2}, 8^{3}, 7^{6}, 6^{4}, 5^{6}, 4^{6}, 3^{4}, 2^{7}, 1^{4}, 0^{3}$ & 46 & 27 & 10 \\
\hline $\begin{array}{l}2000022 \\
0\end{array}$ & $14,10^{9}, 8^{7}, 6,4^{8}, 2,0^{21}$ & 48 & 28 & $\mathrm{~N}$ \\
\hline $\begin{array}{l}0010010 \\
0 \\
\end{array}$ & $9^{2}, 8^{3}, 7^{4}, 6^{6}, 5^{6}, 4^{6}, 3^{8}, 2^{6}, 1^{4}, 0^{3}$ & 48 & 28 & 12 \\
\hline $\begin{array}{l}2000020 \\
0\end{array}$ & $10^{2}, 8^{8}, 6^{8}, 4^{15}, 2^{3}, 0^{14}$ & 50 & 29 & 68 \\
\hline $\begin{array}{l}0000002 \\
2 \\
\end{array}$ & $10,8^{6}, 6^{14}, 4^{7}, 2^{14}, 0^{8}$ & 50 & 29 & 34 \\
\hline $\begin{array}{c}2000101 \\
0\end{array}$ & $10,9^{2}, 8^{7}, 6,5^{14}, 4^{7}, 3^{2}, 2,0^{17}$ & 52 & 30 & 84 \\
\hline $\begin{array}{l}0010002 \\
0\end{array}$ & $10,8^{3}, 7^{6}, 6^{8}, 5^{6}, 4^{3}, 3^{2}, 2^{7}, 1^{10}, 0^{6}$ & 52 & 30 & 16 \\
\hline $\begin{array}{l}0100100 \\
0 \\
\end{array}$ & $8^{3}, 7^{4}, 6^{5}, 5^{6}, 4^{10}, 3^{8}, 2^{7}, 1^{6}, 0^{3}$ & 52 & 30 & 12 \\
\hline $\begin{array}{l}0000200 \\
0\end{array}$ & $8^{3}, 6^{12}, 4^{15}, 2^{18}, 0^{6}$ & 56 & 32 & 28 \\
\hline $\begin{array}{l}0010001 \\
0\end{array}$ & $8,7^{4}, 6^{5}, 5^{8}, 4^{9}, 3^{8}, 2^{9}, 1^{8}, 0^{4}$ & 56 & 32 & 14 \\
\hline $\begin{array}{c}1000102 \\
0\end{array}$ & $10,8,7^{8}, 6^{8}, 5^{8}, 4,2^{8}, 1^{8}, 0^{15}$ & 58 & 33 & 54 \\
\hline $\begin{array}{c}1000101 \\
0\end{array}$ & $8,7^{2}, 6^{7}, 5^{8}, 4^{9}, 3^{8}, 2^{8}, 1^{8}, 0^{9}$ & 60 & 34 & 29 \\
\hline $\begin{array}{l}1001000 \\
0\end{array}$ & $7^{4}, 6^{6}, 5^{4}, 4^{10}, 3^{16}, 2^{6}, 1^{4}, 0^{10}$ & 60 & 34 & 36 \\
\hline $\begin{array}{c}0000012 \\
1\end{array}$ & $8^{2}, 7^{6}, 6^{13}, 5^{6}, 2^{2}, 1^{14}, 0^{21}$ & 64 & 36 & 77 \\
\hline $\begin{array}{l}0000000 \\
2 \\
\end{array}$ & $6^{8}, 4^{20}, 2^{28}, 0^{8}$ & 64 & 36 & 32 \\
\hline $\begin{array}{l}0010000 \\
0\end{array}$ & $6^{5}, 5^{6}, 4^{10}, 3^{14}, 2^{15}, 1^{10}, 0^{6}$ & 66 & 37 & 22 \\
\hline $\begin{array}{l}2000002 \\
0 \\
\end{array}$ & $8,6^{11}, 4^{21}, 2^{11}, 0^{24}$ & 68 & 38 & 90 \\
\hline $\begin{array}{l}1000100 \\
0\end{array}$ & $6^{3}, 5^{8}, 4^{8}, 3^{16}, 2^{16}, 1^{8}, 0^{11}$ & 70 & 39 & 35 \\
\hline
\end{tabular}




\begin{tabular}{|c|c|c|c|c|}
\hline $\begin{array}{l}0000010 \\
1 \\
\end{array}$ & $6^{2}, 5^{6}, 4^{13}, 3^{12}, 2^{16}, 1^{14}, 0^{9}$ & 72 & 40 & 28 \\
\hline $\begin{array}{c}0100001 \\
0 \\
\end{array}$ & $6,5^{6}, 4^{11}, 3^{16}, 2^{15}, 1^{14}, 0^{13}$ & 76 & 42 & 39 \\
\hline $\begin{array}{l}0000022 \\
0 \\
\end{array}$ & $10,6^{26}, 2,0^{52}$ & 80 & 44 & $\mathrm{~N}$ \\
\hline $\begin{array}{l}0001000 \\
0\end{array}$ & $5^{4}, 4^{10}, 3^{16}, 2^{20}, 1^{20}, 0^{10}$ & 80 & 44 & 32 \\
\hline $\begin{array}{l}0000020 \\
0 \\
\end{array}$ & $6^{2}, 4^{25}, 2^{27}, 0^{28}$ & 82 & 45 & 88 \\
\hline $\begin{array}{l}0000101 \\
0\end{array}$ & $6,5^{2}, 4^{15}, 3^{18}, 2^{10}, 1^{14}, 0^{24}$ & 84 & 46 & 68 \\
\hline $\begin{array}{l}1000010 \\
0\end{array}$ & $5^{2}, 4^{10}, 3^{16}, 2^{23}, 1^{18}, 0^{17}$ & 86 & 47 & 48 \\
\hline $\begin{array}{l}2000000 \\
0 \\
\end{array}$ & $4^{14}, 2^{50}, 0^{28}$ & 92 & 50 & 72 \\
\hline $\begin{array}{l}0100000 \\
0 \\
\end{array}$ & $4^{7}, 3^{14}, 2^{28}, 1^{28}, 0^{17}$ & 94 & 51 & 48 \\
\hline $\begin{array}{c}1000002 \\
0 \\
\end{array}$ & $5,4^{12}, 3^{31}, 2,0^{55}$ & 100 & 54 & $\mathbf{N}$ \\
\hline $\begin{array}{l}0000100 \\
0\end{array}$ & $4^{3}, 3^{16}, 2^{27}, 1^{32}, 0^{24}$ & 102 & 55 & 60 \\
\hline $\begin{array}{c}1000001 \\
0\end{array}$ & $4,3^{12}, 2^{32}, 1^{32}, 0^{35}$ & 112 & 60 & 80 \\
\hline $\begin{array}{l}0000000 \\
1\end{array}$ & $3^{8}, 2^{28}, 1^{48}, 0^{36}$ & 120 & 64 & 80 \\
\hline $\begin{array}{c}0000002 \\
0\end{array}$ & $4,2^{55}, 0^{78}$ & 134 & 71 & $\mathbf{N}$ \\
\hline $\begin{array}{l}0000010 \\
0\end{array}$ & $3^{2}, 2^{27}, 1^{52}, 0^{55}$ & 136 & 72 & 108 \\
\hline $\begin{array}{c}1000000 \\
0\end{array}$ & $2^{14}, 1^{64}, 0^{78}$ & 156 & 82 & $\mathrm{~N}$ \\
\hline $\begin{array}{l}0000001 \\
0 \\
\end{array}$ & $2,1^{56}, 0^{133}$ & 190 & 99 & $\mathbf{N}$ \\
\hline $\begin{array}{l}0000000 \\
0 \\
\end{array}$ & $0^{248}$ & 248 & 128 & $\mathbf{N}$ \\
\hline
\end{tabular}

Table IV: $\left(\mathfrak{f}_{4}, \mathfrak{a p}(3, \mathrm{C})+\mathfrak{b l}(2, \mathrm{C})\right)$

\begin{tabular}{lllll}
\hline$\Delta(X)$ & $n_{1}, \cdots, n_{p}$ & $p$ & $q$ & $n$ \\
\hline
\end{tabular}




\begin{tabular}{|c|c|c|c|c|}
\hline $22 \Rightarrow 22$ & $22,14,10,2$ & 4 & 4 & 28 \\
\hline $22 \Rightarrow 02$ & $14,10,10,6,4,2$ & 6 & 5 & 14 \\
\hline $02 \Rightarrow 02$ & $10,10,8,6,4,2^{3}$ & 8 & 6 & 10 \\
\hline $10 \Rightarrow 12$ & $10,9,9,6,3,3,2,0^{3}$ & 10 & 7 & 28 \\
\hline $22 \Rightarrow 00$ & $10,6^{5}, 2,0^{3}$ & 10 & 7 & 28 \\
\hline $02 \Rightarrow 00$ & $6^{2}, 4^{4}, 2^{6}$ & 12 & 8 & 8 \\
\hline $10 \Rightarrow 10$ & $6,5^{2}, 4,3^{4}, 2^{3}, 0^{3}$ & 14 & 9 & 17 \\
\hline $01 \Rightarrow 01$ & $5^{2}, 4^{3}, 3^{2}, 2^{2}, 1^{4}, 0^{3}$ & 16 & 10 & 14 \\
\hline $20 \Rightarrow 01$ & $6,4^{4}, 3^{4}, 2,0^{6}$ & 16 & 10 & 28 \\
\hline $00 \Rightarrow 10$ & $4^{3}, 3^{2}, 2^{6}, 1^{4}, 0^{3}$ & 18 & 11 & 13 \\
\hline $00 \Rightarrow 02$ & $4^{7}, 2,0^{14}$ & 22 & 13 & $\mathbf{N}$ \\
\hline $20 \Rightarrow 00$ & $4,2^{13}, 0^{8}$ & 22 & 13 & 24 \\
\hline $01 \Rightarrow 00$ & $3^{2}, 2^{6}, 1^{10}, 0^{6}$ & 24 & 14 & 18 \\
\hline $00 \Rightarrow 01$ & $2^{7}, 1^{8}, 0^{15}$ & 30 & 17 & $\mathrm{~N}$ \\
\hline $10 \Rightarrow 00$ & $2,1^{14}, 0^{21}$ & 36 & 20 & $\mathrm{~N}$ \\
\hline $00 \Rightarrow 00$ & $0^{52}$ & 52 & 28 & $\mathbf{N}$ \\
\hline
\end{tabular}

Table V: $\left(\mathfrak{g}_{2}, \operatorname{sll}(2, \mathbf{C})+\mathfrak{g l}(2, \mathbf{C})\right)$

\begin{tabular}{llllll}
\hline$\Delta(X)$ & \multicolumn{1}{c}{$n_{1}, \cdots, n_{p}$} & $p$ & $q$ & $n$ \\
\hline $2 \Rightarrow 2$ & 10,2 & 2 & 2 & 8 \\
\hline $2 \Rightarrow 0$ & $4,2,2,2$ & 4 & 3 & 4 \\
\hline $0 \Rightarrow 1$ & $3,3,2,0,0,0$ & 6 & 4 & $\mathrm{~N}$ \\
\hline $1 \Rightarrow 0$ & $2,1,1,1,1,0,0,0$ & 8 & 5 & 8 \\
\hline $0 \Rightarrow 0$ & $0^{14}$ & 14 & 8 & $\mathrm{~N}$ \\
\hline
\end{tabular}




\section{References}

[A] Atiyah, M. F., Characters of semi-simple Lie groups (lectures given in Oxford), Mathematical Institute, Oxford, 1976.

[D] Dynkin, E. B., Semisimple subalgebras of semisimple Lie algebras, Amer. Math. Soc. Translations Ser. 2, 6 (1957), 111-245.

[E] Elashvili, A. G., The centralizers of nilpotent elements in the semisimple Lie algebras, Mat. Inst. Razmadze Akad. SSR, 46 (1975), 109-132.

[El] Elkington, G. B., Centralizers of unipotent elements in semisimple algebraic groups, J. Algebra, 23 (1972) 137-163.

[HC1] Harish-Chandra, Invariant eigendistributions on semisimple Lie groups, Bull. Amer. Math. Soc., 69 (1963), 117-123.

[HC2] - Invariant distributions on Lie algebras, Amer. J. Math., 86 (1964), 271-309.

[HC3] - Invariant differential operators and distributions on a semisimple Lie algebra, Amer. J. Math., 86 (1964), 534-564.

[He] Helgason, S., Differential Geometry, Lie Groups, and Symmetric Spaces, Academic Press, 1978.

[HK] Hotta, R. and Kashiwara, M., Invariant holonomic systems on semisimple Lie algebras, Invent. math., 75 (1984), 327-358.

[K] Kengmana, T., Characters of the discrete series for pseudo-Riemannian symmetric spaces, in "Representation theory of reductive groups", edited by P. C. Trombi, Birkhäuser, 1983, 177-183.

[KR] Kostant, B. and Rallis, S., Orbits and representations associated with symmetric spaces, Amer. J. Math., 93 (1971), 753-809.

[O] Oshima, T., A definition of boundary values of solutions of partial differential equations with regular singularities, Publ. RIMS, Kyoto Univ., 19 (1983), 1203-1230.

[OM] Oshima, T. and Matsuki, T., Orbits on affine symmetric spaces under the action of the isotropy subgroups, J. Math. Soc. Japan, 32 (1980), 399-414.

[OS] Oshima, T. and Sekiguchi, J., The restricted root system of a semisimple symmetric pair, Advanced Studies in Pure Math., 4 (1984), 433-497.

[S] Sekiguchi, J., The nilpotent subvariety of the vector space associated to a symmetric pair, Publ. RIMS, Kyoto Univ., 20 (1984), 155-212.

[SKK] Sato, M., Kawai, T. and Kashiwara, M., Microfunctions and pseudo-differential equations, in "Hyperfunctions and pseudo-differential equations", Lecture Notes in Math., 287, Springer, Berlin-Heidelberg-New York, 1973, 265-529.

[V] Varadarajan, V. S., Harmonic analysis on real reductive groups, Lecture notes in Math., 576, Springer, Berlin-Heidelberg-New York, 1977.

[vD] van Dijk, G., Invariant eigendistributions on the tangent space of a rank one semisimple symmetric space, Math. Ann., 268 (1984), 405-416.

[W] Warner, G., Harmonic Analysis on Semi-Simple Lie Groups I, Springer Verlag, 1972.

Department of Mathematics

Tokyo Metropolitan University

Fukasawa, Setagaya

Tokyo, 158 Japan 